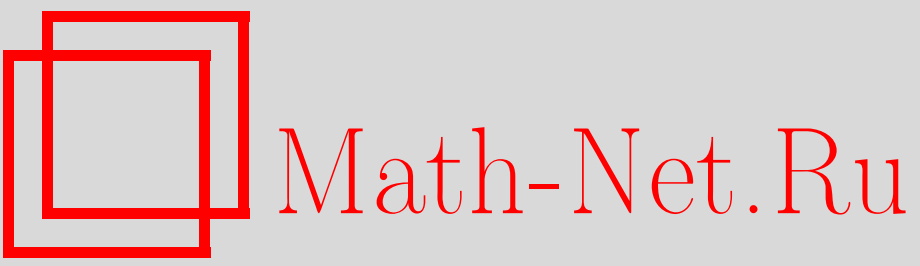

А. И. Оксак, А. Д. Суханов, Представление квантового броуновского движения в методе коллективной координаты, ТМФ, 2003, том 136, номер 1, 115-147

DOI: https://doi.org/10.4213/tmf213

Использование Общероссийского математического портала Math-Net.Ru подразумевает, что вы прочитали и согласны с пользовательским соглашением

http://www.mathnet.ru/rus/agreement

Параметры загрузки:

IP: 52.87 .193 .239

26 апреля 2023 г., 17:34:06 


\section{ПРЕДСТАВЛЕНИЕ КВАНТОВОГО БРОУНОВСКОГО ДВИЖЕНИЯ В МЕТОДЕ КОЛЛЕКТИВНОЙ КООРДИНАТЫ}

Рассматриваются две явно решаемые модели квантовых случайных процессов, описываемых уравнением Ланжевена, - "свободная" квантовая броуновская частица и квантовый броуновский гармонический осциллятор. Гамильтонова (струнная) реализация моделей выявляет солитоноподобную структуру "классических" решений. Соответственно метод коллективной координаты нулевой моды оказывается адекватным средством описания квантовой динамики моделей.

Ключевые слова: квантовое уравнение Ланжевена, струнная модель термостата, температурные представления, асимптотические свойства ковариации.

\section{1. ВВЕДЕНИЕ}

Модели броуновского движения относятся к простейшим типам термодинамически неравновесных процессов. Они играют сушественную роль при описании квантовых, в частности, низкотемпературных явлений - электронных, оптических и др. [1]-[5]. Для конкретности мы будем иметь в виду механические системы. Термостат, с которым взаимодействует броуновская частица, можно считать бесконечной системой. Поэтому отклонения от равновесного теплового состояния носят локальный характер, так что броуновский процесс протекает на фоне равновесного состояния с определенной температурой.

Для последовательной квантовой теории броуновского движения целесообразно иметь дело с гамильтоновой системой. В статистической физике традиционно допускается, что термостат можно моделировать бесконечным набором осцилляторов. В этой связи важное значение имеет работа [6], где было показано, что уравнение Ланжевена может быть смоделировано в рамках некоторой гамильтоновой системы с бесконечным числом степеней свободы. В классическом случае такая имитация броуновского движения позволяет полностью воспроизвести результаты теории Орнштейна-Уленбека для

\footnotetext{
* Российский заочный институт текстильной и легкой промышленности, Москва, Россия

${ }^{\dagger}$ Российский университет дружбы народов, Москва, Россия. E-mail: ogol@mx.pfu.edu.ru; ogol@oldi.ru
} 
уравнения Ланжевена. Применяя далее известные методы квантования гамильтоновой системы, можно построить квантовые аналоги броуновской частицы и уравнения Ланжевена. В работах [7], [8] описан усовершенствованный способ гамильтоновой имитации броуновского движения с помощюю модели Лэмба [9].

Модель Лэмба особенно наглядна, если в качестве независимой переменной выбрано входящее, или in-поле $\varphi^{\text {in }}(t+x)$, представляющее собой свободную волну произвольной конфигурации, перемещающуюся с постоянной скоростью против направления координатной оси. Энергетические соображения допускают произвольные значения $\varphi^{\text {in }}( \pm \infty)$ солитоноподобных (или кинкоподобных) конфигураций in-поля. Разность этих значений с коэффишиентом $1 / \sqrt{2}$ является топологическим зарядом $Z$ - сохраняющейся величиной. Это величина непрерывна, в отличие от топологического заряда в одномерных полевых теориях с обычными солитонными решениями (кинками); в них топологический заряд дискретен вследствие энергетических ограничений.

Мы рассматриваем две явно решаемые модели одномерного квантового броуновского движения - для “свободной” частицы (разделы 2-6) и для гармонического осциллятора (раздел 7). Имеются явные формулы, сводящие случай осциллятора к "свободной” частице, поэтому основное внимание уделено “свободной” броуновской частице. В струнной реализации система представляет собой квантово-полевую линейную бозонную модель, точнее, левую составляющую скалярного поля нулевой массы в одномерном пространстве. Наличие солитоноподобных решений классического поля проявляется при квантовании в инфракрасных расходимостях формальной двухточечной функции. Вследствие этого данная линейная бозонная система не является фоковской: в системе отсутствуют равновесные состояния с определенной температурой, вместо них появляются равновесные обобшенные состояния с бесконечной нормой. Как показано в работе [10], такое обобшенное состояние позволяет построить температурное представление (с температурой $T>0$ ); ранее в работе [11] было построено вакуумное представление, соответствующее нулевой температуре (см. также [12], гл.11). Модель подчеркивает необходимость иметь дело с неравновесными состояниями представления наблюдаемых в гильбертовом пространстве, а не с одним избранным равновесным состоянием (ввиду отсутствия такового).

Топологический заряд $Z$ является также генератором калибровочных преобразований струнного поля, которые, в свою очередь, сводятся к пространственным трансляциям имитируемой системы частища-термостат. Поэтому $Z$ отличается лишь постоянным множителем от полного импульса $K$ системы (сохраняющейся величины). Наличие такой симметрии вместе с солитоноподобной структурой фазового пространства классических решений указывает на возможность квантования модели методом коллективной координаты. Вообще, коллективной координатой называют динамическую переменную с одной степенью свободы, сконструированную из полного набора динамических переменных, причем она (или они, если их несколько) подчиняется определенному закону при преобразованиях данной симметрии. Остальные динамические переменные, дополняюшие коллективную (или коллективные) до полного набора, должны быть инвариантами преобразований симметрии. 
В данной работе мы используем коллективную координату нулевой моды, связанную с калибровочной симметрией группы $\mathbb{R}$ (см. [13], п. 8.2). В этом варианте новый набор переменных явно выражается через исходные переменные. Координата $q(t)$ броуновской частицы разбивается в сумму нефоковской солитоноподобной составляющей - коллективной переменной $q^{\mathrm{col}}(t)$ и фоковской чисто фононной составляющей $q^{(0)}(t)$. Такое разбиение приводит к альтернативному способу построения температурных представлений, проясняющему их структуру. При подходящем выборе коллективной координаты полное гильбертово пространство $\mathcal{H}$ температурного представления можно преобразовать в тензорное произведение $\mathcal{L}^{2}(\mathbb{R}) \otimes \mathcal{H}^{(0)}$ пространства представления калибровочно-неинвариантной составляющей $q^{\mathrm{col}}(t)$ и пространства гиббсовского представления чисто фононной калибровочно-инвариантной составляющей $q^{(0)}(t)$. Указанное разбиение для $q(t)$ удобно также при изучении динамики броуновского движения при больших временах.

Раздел 2 работы содержит основные соотношения гамильтоновой модели "свободной” броуновской частицы. В разделе 3 приведена явно ковариантная конструкция температурных представлений для этой модели. Основная проблема использования коллективной координаты связана с доказательством того, что при подходящем определении $q^{\mathrm{col}}(t)$ представление бозонной системы в тензорном произведении $\mathcal{L}^{2}(\mathbb{R}) \otimes \mathcal{H}^{(0)}$ унитарно-эквивалентно построенному в разделе 3 температурному представлению. В связи с этим в разделах 4 и 5 вместо определения ad hoc мы используем конструктивный метод определения $q^{\mathrm{col}}(t)$, приводя пространство представления $\mathcal{H}$ к указанному тензорному произведению. В замечании в разделе 5 сфформулирован набор характеристических условий, который позволяет определить “подходящую" коллективную координату $q^{\mathrm{col}}(t)$ и произвести однозначное разбиение $q(t)$ в сумму $q^{\mathrm{col}}(t)$ и калибровочно-инвариантной составляющей $q^{(0)}(t)$. В разделе 6 мы используем полученные разложения при рассмотрении асимптотических свойств ковариаций $q(t)$ и импульса $p(t)$ при больших временах, а также поведения ковариаций при малых $|t|$.

В разделе 7 рассматривается схема квантования броуновского гармонического осциллятора. В работе [7] была установлена связь этой модели с гамильтоновой струнной моделью и построено гиббсовское представление по равновесному состоянию с определенной температурой. Наше изложение модели отличается тем, что использование в полной мере струнной реализации позволяет включить в рассмотрение более широкий класс неравновесных состояний, а именно состояния с ненулевым топологическим зарядом $Z$ (состояния гиббсовского представления из работы [7] находятся в "нулевом сектоpe" $Z=0)$. С помощью модели “свободной” броуновской частицы с координатой $q(t)$ и импульсом $p(t)$ можно исследовать модель броуновского осциллятора: после необходимых отождествлений координата $Q(t)$ осциллятора выражается через $q(t)$ или $p(t)$. При этом полный гамильтониан $H$ и топологический заряд $Z$ обеих систем в соответствующих температурных представлениях одинаковы (после упомянутых отождествлений).

Перечислим обозначения, часто встречающиеся в дальнейшем. $\mathbb{R}$ - вещественная прямая, $\overline{\mathbb{R}}_{+}=\{x \in \mathbb{R}: x \geqslant 0\}, \overline{\mathbb{R}}_{-}=\{x \in \mathbb{R}: x \leqslant 0\}$. Через $\mathcal{S}_{\mathrm{r}}(\mathbb{R})$ и $\mathcal{S}_{\mathrm{r}}\left(\overline{\mathbb{R}}_{ \pm}\right)$мы обозначаем подпространства всех вешественных основных функций в пространствах Швар- 
ца $\mathcal{S}(\mathbb{R})$ и $\mathcal{S}\left(\mathbb{R}_{ \pm}\right.$) (обычно мы пишем $f(t) \in \mathcal{S}_{\mathrm{r}}(\mathbb{R})$ вместо $f \in \mathcal{S}_{\mathrm{r}}(\mathbb{R})$ по $t$ ). Через $\mathcal{S}^{\prime}(\mathbb{R})$ обозначается пространство Шварца обобшенных функций умеренного роста на $\mathbb{R}$. Под $\varepsilon * \mathcal{S}_{\mathrm{r}}(\mathbb{R})$ подразумевается множество всех $\mathcal{C}^{\infty}$-функций типа свертки $\varepsilon(t) * g(t)$ при $g(t) \in \mathcal{S}_{\mathrm{r}}(\mathbb{R})$, где $\varepsilon(t)=1$ при $t \geqslant 0$ и $\varepsilon(t)=-1$ при $t<0$. Преобразование Фурье в пространствах $\mathcal{S}(\mathbb{R})$ и $\mathcal{S}^{\prime}(\mathbb{R})$ записьваем в виде

$$
\tilde{f}(\lambda)=\int e^{i \lambda t} f(t) d t
$$

При $k \in \mathbb{R}$ обозначаем пространство

$$
\mathcal{F}^{(k)}=\left\{f \in \mathcal{S}_{\mathrm{r}}(\mathbb{R}): \tilde{f}(0) \equiv \int f(t) d t=k\right\} .
$$

Пространство $\mathcal{A}^{(k)}$ определяется далее в разделе 4 .

\section{2. ГАМИЛЬТОНОВА МОДЕЛЬ “СВОБОДНОЙ" БРОУНОВСКОЙ ЧАСТИЦЫ}

Уравнение Ланжевена для “свободной” броуновской частицы массы $m$, совершаюшей одномерное движение, имеет вид

$$
m\left(\partial_{t}+\gamma\right) \partial_{t} q(t)=\sqrt{2 \gamma m} \xi(t) .
$$

Здесь $q(t)$ - координата броуновской частицы в момент $t, m \partial_{t} q(t)=p(t)$ - импульс частицы, $m \gamma>0$ - коэффициент вязкого трения, $-m \gamma \partial_{t} q(t)$ и $\sqrt{2 \gamma m} \xi(t)-$ соответственно диссипативная и флуктуируюшая (ведушая) силы, действуюшие на частицу.

В квантовом случае все содержание модели может быть выведено из разновременных коммутационных соотношений

$$
\left[q\left(t_{1}\right), q\left(t_{2}\right)\right]=\frac{1}{i} D\left(t_{1}-t_{2}\right)
$$

"координатного поля" $q(t)$, представляющего собой эрмитову операторную обобщенную функцию над пространством Шварца основных функций $\mathcal{S}(\mathbb{R})$, действуюшую в некотором гильбертовом пространстве $\mathcal{H}$ векторов состояний ${ }^{1)}$. Перестановочная функция

$$
D(t)=\frac{2 \gamma i}{m} \int \frac{1}{\gamma^{2}+\lambda^{2}}\left(P \frac{1}{\lambda}\right) e^{-i \lambda t} \frac{d \lambda}{2 \pi}=\frac{\gamma}{m} \cdot \frac{1}{\gamma^{2}-\partial_{t}^{2}} \varepsilon(t)=\frac{1}{\gamma m}\left(1-e^{-\gamma|t|}\right) \varepsilon(t)
$$

есть единственное решение уравнения

$$
\left(\gamma^{2}-\partial_{t}^{2}\right) D(t)=\frac{\gamma}{m} \varepsilon(t)
$$

\footnotetext{
1) Здесь и далее полагаем $\hbar=1$.
} 
в классе $\mathcal{S}^{\prime}(\mathbb{R})$.

Разновременные коммутационные соотношения для "импульсного поля" $p(t)$ имеют вид

$$
\begin{aligned}
& {\left[q\left(t_{1}\right), p\left(t_{2}\right)\right]=-\frac{m}{i} \partial_{t_{1}} D\left(t_{1}-t_{2}\right)=i e^{-\gamma\left|t_{1}-t_{2}\right|},} \\
& {\left[p\left(t_{1}\right), p\left(t_{2}\right)\right]=-\frac{m^{2}}{i} \partial_{t_{1}}^{2} D\left(t_{1}-t_{2}\right)=-\gamma m i e^{-\gamma\left|t_{1}-t_{2}\right|} \varepsilon\left(t_{1}-t_{2}\right) .}
\end{aligned}
$$

После введения канонических коммутационных соотношений (KKC) для поля $q(t)$ возникает задача построения наиболее интересных физических представлений $\mathrm{KKC}$, а именно температурных $(T>0)$ и вакуумного, являющегося предельным случаем $(T=0)$ температурных представлений. Как следует из дальнейшего изложения, термодинамически равновесные состояния сушествуют для импульсного, но не для координатного поля, и температурные представления для $q(t)$ приводят к необходимости рассматривать вместо равновесных близкие к равновесным состояния. Отметим, что в приведенной постановке задачи роль уравнения Ланжевена сводится к определяюшему соотношению для флуктуируюшей силы $\sqrt{2 \gamma m} \xi(t)$.

Приведем необходимое пояснение относительно происхождения KKC (3). Как указано во введении, для целей квантования подбирается подходящая гамильтонова реализация уравнений Ланжевена. В соответствии с работами [7], [8] система "частица+термостат" моделируется с использованием струны Лэмба [9]. Роль термостата играет полубесконечная струна, натянутая вдоль полуоси $O x_{+}$. Струна совершает колебания в направлении оси $O y$. "Свободная" броуновская частица имитируется точечной массивной частицей массы $m$, жестко прикрепленной к концу $(x=0)$ струны, который может свободно скользить вдоль оси $O y$. В результате частица движется по оси $O y$. Конфигурацию струны в момент $t \in \mathbb{R}$ обозначим через

$$
y=(\gamma m)^{-1 / 2} \varphi(t, x), \quad x \geqslant 0,
$$

где $\varphi(t, x)$ можно назвать струнным полем. Соответственно

$$
q(t)=(\gamma m)^{-1 / 2} \varphi(t, 0)
$$

есть координата частишы на оси $O y$ в момент $t$.

Лагранжиан системы есть сумма лагранжиана свободной полубесконечной струны и кинетической энергии частишы,

$$
L=\frac{1}{2} \int\left(\left(\partial_{t} \varphi\right)^{2}-\left(\partial_{x} \varphi\right)^{2}\right) \theta(x) d x+\frac{1}{2 \gamma} \int\left(\partial_{t} \varphi\right)^{2} \delta(x) d x .
$$

Здесь $\theta(x)=1$ при $x \geqslant 0, \theta(x)=0$ при $x<0$. "Канонический импульс", сопряженный c "канонической координатой" $\varphi(t, x)$, есть

$$
j(t, x)=\frac{\delta L}{\delta \partial_{t} \varphi}=\partial_{t} \varphi\left(\theta(x)+\gamma^{-1} \delta(x)\right) .
$$


Гамильтониан системы

$$
H=\int j(t, x) \partial_{t} \varphi(t, x) d x-L=\frac{1}{2} \int\left(\left(\partial_{t} \varphi\right)^{2}+\left(\partial_{x} \varphi\right)^{2}\right) \theta(x) d x+\frac{1}{2 \gamma} \int\left(\partial_{t} \varphi\right)^{2} \delta(x) d x,
$$

а уравнение движения $\partial_{t} j-\delta L / \delta \varphi=0$ означает

$$
\left(\partial_{t}^{2}-\partial_{x}^{2}\right) \varphi \theta(x)+\left(\gamma^{-1} \partial_{t}^{2}-\partial_{x}\right) \varphi \delta(x)=0 .
$$

Оно сводится к волновому уравнению $\left(\partial_{t}^{2}-\partial_{x}^{2}\right) \varphi \theta(x)=0$ и граничному условию $\left(\partial_{t}^{2}-\gamma \partial_{x} \varphi\right) \delta(x)=0$ при $x=0$.

Решение волнового уравнения можно записать в виде

$$
\varphi(t, x)=\frac{1}{\sqrt{2}}\left(\varphi^{\text {in }}(t+x)+\varphi^{\text {out }}(t-x)\right)
$$

где $\varphi^{\text {in }}(t)$ и $\varphi^{\text {out }}(t)$ - одномерные входящее и выходящееполя ( “до” и “после” рассеяния), $t$ - аналог переменной светового (в данном случае звукового) поля. Относительно фазового пространства $\mathcal{X}$ допустимых конфигураций классического струнного поля $\varphi(t, x)$ сделаем предположение, что это гладкие (класса $\mathcal{C}^{\infty}$ ) функции от $(t, x) \in \mathbb{R} \times \overline{\mathbb{R}}_{+}$такие, что $\varphi(t, x)$ и $\partial_{t} \varphi(t, x)$ принадлежат классу $\mathcal{S}_{\mathrm{r}}\left(\overline{\mathbb{R}}_{+}\right)$по $x$ при любом $t \in \mathbb{R}$.

Фазовое пространство $\mathcal{X}$ есть симплектическое пространство с приведенной далее симплектической формой $\sigma^{2)}$. Граничное условие при $x=0$ для волнового уравнения связывает входящее и выходящее поля. Учитывая, что при прибавлении к $\varphi^{\text {in }}(t)$ произвольной константы и вычитания ее из $\varphi^{\text {out }}(t)$ поле $\varphi(t, x)$ не меняется, можно так выбрать эту константу (в зависимости от $\varphi$ ), что связь между $\varphi^{\text {in }}(t)$ и $\varphi^{\text {out }}(t)$ примет вид

$$
\varphi^{\mathrm{out}}(t)=\frac{\gamma-\partial_{t}}{\gamma+\partial_{t}} \varphi^{\mathrm{in}}(t)
$$

Соответственно для координатного поля имеем

$$
q(t)=\left(\frac{2 \gamma}{m}\right)^{1 / 2} \frac{1}{\gamma+\partial_{t}} \varphi^{\text {in }}(t)
$$

Мы можем принять входяшее поле в качестве способа "параметризации" фазового пространства. При этом $\varphi^{\text {in }}(t)$ может быть любой функцией класса $\varepsilon * \mathcal{S}_{\mathrm{r}}(\mathbb{R})$, определенного в разделе 1 (по поводу доказательства см. раздел 2 в работе [10]). K этому же классу принадлежат функции $\varphi^{\text {out }}(t)$ и $q(t)$. Из (11) следует, что для выполнимости

\footnotetext{
${ }^{2)}$ В данном случае бесконечномерного пространства $\mathcal{X}$ имеет смысл различать $\mathcal{X}$ и некоторое его расширение $\widetilde{\mathcal{X}}$, например, наложением условия $\partial_{x} \varphi(t, x) \in \mathcal{S}_{\mathrm{r}}\left(\overline{\mathbb{R}}_{+}\right)$по $x$ вместо прежнего условия $\varphi(t, x) \in \mathcal{S}_{\mathrm{r}}\left(\overline{\mathbb{R}}_{+}\right)$. $\widetilde{\mathcal{X}}$ рассматривается как двойственное пространство к $\mathcal{X}$ относительно билинейной формы $\sigma$. Полевая алгебра классического поля состоит из функционалов от $\varphi \in \tilde{\mathcal{X}}$. Различие между $\mathcal{X}$ и $\widetilde{\mathcal{X}}$ проявляется, например, в вопросе об асимптотике $\varphi^{\text {in }}(t+x)$ при $x \rightarrow \pm \infty$, а также при рассмотрении калибровочных преобразований типа (19) (см. ниже), которые оставляют инвариантным $\widetilde{\mathcal{X}}$.
} 
уравнения Ланжевена $(2)$ поле $\xi(t)$ во флуктуируюшей силе $\sqrt{2 \gamma m} \cdot \xi(t)$ следует отождествить с входяшим током $j^{\text {in }}(t)$,

$$
\xi(t)=j^{\text {in }}(t) \equiv \partial_{t} \varphi^{\text {in }}(t) .
$$

Интересно, что хотя струнное поле $\varphi(t, x)$ первоначально было определено лишь при $x \geqslant 0$, формула (9) осушествляет "принудительное" продолжение его до поля класса $\mathcal{C}^{\infty}$ при всех $x \in \mathbb{R}$, удовлетворяюшего волновому уравнению

$$
\left(\partial_{t}^{2}-\partial_{x}^{2}\right) \varphi(t, x)=0
$$

при всех $(t, x) \in \mathbb{R}^{2}$. Граничное условие при $x=0$ заменяется связью (10).

На фазовом пространстве $\mathcal{X}$ как на симплектическом пространстве задана симплектическая форма $\sigma$, сопоставляюшая каждой паре "классических" решений уравнений движения выражение

$$
\begin{aligned}
\sigma\left(\varphi_{1}, \varphi_{2}\right) & =\int\left(j_{1}(t, x) \varphi_{2}(t, x)-j_{2}(t, x) \varphi_{1}(t, x)\right) d x= \\
& =\int\left(\left(\partial_{t} \varphi_{1}\right) \varphi_{2}-\varphi_{1} \partial_{t} \varphi_{2}\right)\left(\theta(x)+\gamma^{-1} \delta(x)\right) d x .
\end{aligned}
$$

Подставив сюда разложение (9) и совершив предельный переход $t \rightarrow-\infty$, получим выражение для симплектической формы в терминах $\varphi_{1}^{\text {in }}, \varphi_{2}^{\text {in }}$ :

$$
\sigma\left(\varphi_{1}, \varphi_{2}\right)=\frac{1}{2} \int\left(\left(\partial_{t} \varphi_{1}^{\mathrm{in}}(t)\right) \varphi_{2}^{\mathrm{in}}(t)-\varphi_{1}^{\mathrm{in}}(t) \partial_{t} \varphi_{2}^{\mathrm{in}}(t)\right) d t .
$$

Скобки Пуассона "классического" поля $\varphi(t, x)$ можно найти исходя из стандартного соотношения

$$
\left\{\sigma\left(\varphi, \varphi_{1}\right), \sigma\left(\varphi, \varphi_{2}\right)\right\}=\sigma\left(\varphi_{1}, \varphi_{2}\right),
$$

где $\varphi_{1}, \varphi_{2} \in \mathcal{X}, \varphi \in \tilde{\mathcal{X}}$.

Если $\varphi_{j}^{\text {in }}$ выбрать в виде $\varphi_{j}^{\text {in }}=\frac{1}{2} \varepsilon * f_{j}, f_{j} \in \mathcal{S}_{\mathrm{r}}(\mathbb{R}), j=1,2$, то

$$
\int \varphi^{\mathrm{in}}(t) f_{j}(t) d t=-\sigma\left(\varphi, \varphi_{j}\right)
$$

и мы получаем

$$
\begin{aligned}
& \left\{\int \varphi^{\mathrm{in}}\left(t_{1}\right) f_{1}\left(t_{1}\right) d t_{1}, \int \varphi^{\mathrm{in}}\left(t_{2}\right) f_{2}\left(t_{2}\right) d t_{2}\right\}=\sigma\left(\varphi_{1}, \varphi_{2}\right)= \\
& =\frac{1}{2} \int \varepsilon\left(t_{1}-t_{2}\right) f_{1}\left(t_{1}\right) f_{2}\left(t_{2}\right) d t_{1} d t_{2} .
\end{aligned}
$$

Следовательно, разновременные скобки Пуассона поля $\varphi^{\text {in }}(t)$ имеют вид

$$
\left\{\varphi^{\mathrm{in}}\left(t_{1}\right), \varphi^{\mathrm{in}}\left(t_{2}\right)\right\}_{P}=D^{\mathrm{in}}\left(t_{1}-t_{2}\right),
$$


где

$$
D^{\text {in }}(t)=i \int\left(P \frac{1}{\lambda}\right) e^{-i \lambda t} \frac{d \lambda}{2 \pi}=\frac{1}{2} \varepsilon(t) .
$$

При квантовании скобки Пуассона заменяются коммутатором для входящего поля

$$
\left[\varphi^{\text {in }}\left(t_{1}\right), \varphi^{\text {in }}\left(t_{2}\right)\right]=\frac{1}{i} D^{\text {in }}\left(t_{1}-t_{2}\right)
$$

откуда следуют приведенные ранее $\mathrm{KKC}(3),(5),(6)$ для координатного и импульсного полей.

Рассмотрим вопрос о симметриях в рамках модели классических полей. Инвариантность лагранжиана относительно однопараметрической группы трансляций по времени, при которых

$$
\varphi(t, x) \rightarrow \varphi(t-\tau, x), \quad q(t) \rightarrow q(t-\tau), \quad p(t) \rightarrow p(t-\tau), \quad \tau \in \mathbb{R},
$$

как известно, имеет следствием то, что гамильтониан $H$ системы сохраняется. Он связан с входящим током $j^{\text {in }}(t)$ (функцией класса $\mathcal{S}_{\mathrm{r}}(\mathbb{R})$ ) соотношением

$$
H=\frac{1}{2} \int\left(j^{\text {in }}(t)\right)^{2} d t
$$

Особую роль в нашем изложении играет инвариантность лагранжиана или гамильтониана струнного поля относительно однопараметрической групшы калибровочных преобразований первого рода

$$
\varphi(t, x) \rightarrow \varphi(t, x)+g, \quad g \in \mathbb{R}
$$

при которых

$$
\varphi^{\text {in }}(t) \rightarrow \varphi^{\text {in }}(t)+\frac{1}{\sqrt{2}} g, \quad \varphi^{\text {out }}(t) \rightarrow \varphi^{\text {out }}(t)+\frac{1}{\sqrt{2}} g .
$$

Интеграл по $x$ от временной компоненты $j(t, x)$ "сохраняющегося" нетеровского тока есть сохраняющаяся величина, выражаемая через $j^{\text {in }}(t)$ по формуле

$$
Z=\frac{1}{\sqrt{2}} \int j^{\text {in }}(t) d t
$$

Так как $Z=\left(\varphi^{\text {in }}(+\infty)-\varphi^{\text {in }}(-\infty)\right) / \sqrt{2}$, то величина $Z$ имеет смысл топологического заряда солитоноподобных конфигураций входяшего поля.

Для физической интерпретации полученного заряда отметим, что калибровочная симметрия струнного поля есть, в сушности, симметрия струнной модели относительно трансляций вдоль оси $О y$. Она означает, что имитируемая система “частица+термостат" обладает инвариантностью относительно пространственных трансляций, при которых

$$
q(t) \rightarrow q(t)+r, \quad p(t) \rightarrow p(t)
$$


где роль группового параметра играет $r=(\gamma m)^{-1 / 2} g$ с размерностью координаты. Соответственно сохраняющуюся величину

$$
K=(\gamma m)^{1 / 2} Z=\left(\frac{\gamma m}{2}\right)^{1 / 2} \int \xi(t) d t
$$

следует назвать полным импульсом системы “частица+термостат". Заметим, что $2 K$ имеет смысл импульса, переданного частице флуктуируюшей силой за время от $-\infty$ до $+\infty$. Из уравнений Ланжевена $(2)$ с учетом того, что $p(t) \in \mathcal{S}_{\mathrm{r}}(\mathbb{R})$, следует также формула

$$
K=\frac{\gamma}{2} \int p(t) d t
$$

Это означает, что $-2 K$ есть импульс, переданный частице силой вязкого трения за время от $-\infty$ до $+\infty$.

\section{3. ТЕМПЕРАТУРНЫЕ ПРЕДСТАВЛЕНИЯ}

3.1. Алгебра $\mathcal{A}$ KКС системы. Рассмотрим конструкцию температурных представлений KKC (3), отсылая за деталями к работе [10]. Абстрактная алгебра ККС $\mathcal{A}$ состоит из линейных комбинаций унитарных элементов $\mathcal{E}_{f} \equiv \mathcal{E}_{-f}^{*}, f \in \mathcal{S}_{\mathrm{r}}(\mathbb{R})$, соответствующих “конкретным” экспонентам

$$
E_{f}=\pi\left(\mathcal{E}_{f}\right)=e^{i q_{f}}
$$

которые появляются при переходе к представлению $\pi$. Здесь $q(t)$ - операторная обобшенная функция над пространством $\mathcal{S}(\mathbb{R})$, а

$$
q_{f} \equiv \int q(t) f(t) d t, \quad p_{g}(t) \equiv \int p(t) g(t) d t, \quad f, g \in \mathcal{S}_{\mathrm{r}}(\mathbb{R})
$$

- эрмитовы операторы в пространстве представления (в рассматриваемых представлениях они имеют общую плотную область целых аналитических векторов). В экспоненциальной форме Вейля KKC (3) записьваются как

$$
\mathcal{E}_{f_{1}} \mathcal{E}_{f_{2}}=e^{-(1 / 2)\left[q_{f_{1}}, q_{f_{2}}\right]} \mathcal{E}_{f_{1}+f_{2}}
$$

где

$$
\left[q_{f_{1}}, q_{f_{2}}\right]=\frac{1}{i} \int D\left(t_{1}-t_{2}\right) f_{1}\left(t_{1}\right) f_{2}\left(t_{2}\right) d t_{1} d t_{2}
$$

- чисто мнимое число. Калибровочным преобразованиям (19), (20) или, что то же, преобразованиям пространственных трансляций $(22)$ соответствуют автоморфизмы $\Gamma_{r}$ алгебры $\mathcal{A}$, при которых

$$
\Gamma_{r}\left(\mathcal{E}_{f}\right)=e^{i \tilde{f}(0) r} \mathcal{E}_{f} \quad \text { при } \quad f \in \mathcal{S}_{\mathrm{r}}(\mathbb{R}), \quad \tilde{f}(0) \equiv \int f(t) d t .
$$


Из определения алгебры $\mathcal{A}$ следует, что она может быть расширена интегралами

$$
A=\int_{\mathcal{M}} \mathcal{E}_{f} d \mu(f),
$$

где $\mathcal{M}$ - произвольное конечномерное подпространство в $\mathcal{S}_{\mathrm{r}}(\mathbb{R})$, а $d \mu(f)$ - произвольная комплексная борелева мера на $\mathcal{M}$ с компактным носителем. При переходе к представлениям этим (отчасти формальным) интегралам соответствуют обычные интегралы $\pi(A)=\int_{\mathcal{M}} E_{f} d \mu(f)$ (в слабой операторной топологии). Меру $d \mu(f)$ в (29) мы называем $\tilde{f}(0)$-гладкой, если для нее имеет место представление в виде

$$
\int_{\mathcal{M}} u(f) d \mu(f)=\sum_{i=1}^{n} \int_{\mathcal{M}_{i}} u(f) F_{i}(f) d l_{i}(f),
$$

где $u(f) \in \mathcal{S}(\mathcal{M})$ по $f, \mathcal{M}_{i}$ - афффинные подпространства в $\mathcal{M}$ с лебеговыми мерами $d l_{i}(f)$, причем образ каждого $\mathcal{M}_{i}$ (а значит, образ $\left.\mathcal{M}\right)$ при отображении $f \rightarrow \tilde{f}(0)$ есть $\mathbb{R} ;$ $F_{i}(f)$ - некоторые функции из $\mathcal{S}(\mathcal{M})$ по $f$. Для $\tilde{f}(0)$-гладких мер $d \mu(f)$ и для $u(f) \in$ $\mathcal{S}(\mathcal{M})$ функция

$$
\int_{\mathcal{M}} u(f) 2 \pi \delta(k-\tilde{f}(0)) d \mu(f)
$$

принадлежит $\mathcal{S}(\mathbb{R})$ по $k$.

Посредством $\pi$ мы будем обозначать температурное представление алгебры $\mathcal{A}$ $\left(T>0\right.$ - температура, $k_{\mathrm{B}}$ - постоянная Больцмана, $\left.\beta=\left(k_{\mathrm{B}} T\right)^{-1}\right)$. Для конструкции $\pi$ удобно использовать формальную двухточечную функцию

$$
\begin{gathered}
\left\langle q\left(t_{1}\right) q\left(t_{2}\right)\right\rangle=W\left(t_{1}-t_{2}\right), \\
W(t)=\frac{2 \gamma}{m} \int\left[\left(\frac{1-e^{-\beta \lambda}}{\lambda}\right)^{-1} P \frac{1}{\lambda^{2}}+a_{T} \delta(\lambda)\right] \frac{e^{-i \lambda t}}{\gamma^{2}+\lambda^{2}} \frac{d \lambda}{2 \pi}= \\
=-\frac{1}{2 \pi m} \ln \left[0+i \varkappa \frac{\beta}{\pi} \operatorname{sh}\left(\frac{\pi t}{\beta}\right)\right] * e^{-\gamma|t|} .
\end{gathered}
$$

Формальная двухточечная функция имеет инфракрасную расходимость (сингулярность при $\lambda=0$ ). При инфракрасной регуляризации (32) появляется вешественньй параметр $a_{T}$ или, что эквивалентно, положительный параметр $\varkappa$ размерности массы. Следствием инфракрасной расходимости является отсутствие положительной определенности формальной двухточечной функции, а также отсутствие на алгебре $\mathcal{A}$ гиббсовского состояния, характеристический функционал которого был бы равен

$$
\left\langle\mathcal{E}_{f}\right\rangle=e^{(-1 / 2)\left\langle\left(q_{f}\right)^{2}\right\rangle} \text { при } f \in \mathcal{S}_{\mathrm{r}}(\mathbb{R}) .
$$

Здесь и далее

$$
\left\langle q_{f_{1}} q_{f_{2}}\right\rangle=\int W\left(t_{1}-t_{2}\right) f_{1}\left(t_{1}\right) f_{2}\left(t_{2}\right) d t_{1} d t_{2}, \quad f_{1}, f_{2} \in \mathcal{S}_{\mathrm{r}}(\mathbb{R}) .
$$

Поэтому выражение (33) есть просто некий формальный функционал. 
3.2. Гиббсовское состояние подалгебры $\mathcal{A}^{(0)}$. Конструкция представления $\pi$ использует свойство условной положительной определенности $W(t)$, а именно $\lambda^{2} \widetilde{W}(\lambda) \geqslant 0$. Пусть $\mathcal{F}^{(0)}$ определено по формуле $(1)$ при $k=0$. Функции $f(t)$ из $\mathcal{F}^{(0)}$ представимы в виде $f(t)=-\partial_{t} g(t)$ при $g(t) \in \mathcal{S}_{\mathrm{r}}(\mathbb{R})$. В силу тождества

$$
q_{-\partial_{t} g}=\frac{1}{m} p_{g} \equiv \frac{1}{m} \int p(t) g(t) d t
$$

подалгебра, порожденная элементами $E_{f}$ при $f \in \mathcal{F}^{(0)}$, является фактически алгеброй импульсного поля $p(t)$. В терминах абстрактной алгебры $\mathcal{A}$ она соответствует подалгебре $\mathcal{A}^{(0)}$ калибровочно-инвариантных (или инвариантных при пространственных трансляциях) элементов $A$,

$$
\Gamma_{r}(A)=A, \quad r \in A \text {. }
$$

Условная положительная определенность $W(t)$ позволяет определить равновесное гиббсовское состояние $s^{(0)}$ подалгебры $\mathcal{A}^{(0)}$ с помощью характеристического функционала

$$
s^{(0)}\left(\mathcal{E}_{f}\right)=e^{(-1 / 2)\left\langle\left(q_{f}\right)^{2}\right\rangle}, \quad f \in \mathcal{F}^{(0)}
$$

Известная конструкция Гельфанда-Наймарка-Сигала (ГНС [14]) позволяет по состоянию $s^{(0)}$ построить представление $\pi^{(0)}$ алгебры $\mathcal{A}^{(0)}$ в некотором гильбертовом пространстве $\mathcal{H}^{(0)}$ с циклическим вектором, который мы обозначим как $|\mathrm{GIBBS}\rangle$. Обозначая посредством $p^{(0)}(t)$ импульсное поле в гиббсовском представлении, имеем, что при $f=-\partial_{t} g \in \mathcal{F}^{(0)}$ выполняются соотношения

$$
\begin{gathered}
\pi^{(0)}\left(\mathcal{E}_{f}\right)=E_{f}^{(0)}=e^{(i / m) p_{g}^{(0)}} \\
\left\langle\mathrm{GIBBS}\left|E_{f}^{(0)}\right| \mathrm{GIBBS}\right\rangle_{0}=e^{\left(-1 / 2 m^{2}\right)\left\langle\left(p_{g}\right)^{2}\right\rangle}, \\
\left\langle\mathrm{GIBBS}\left|p^{(0)}\left(t_{1}\right) p^{(0)}\left(t_{2}\right)\right| \mathrm{GIBBS}\right\rangle_{0}=-m^{2} \partial_{t_{1}}^{2} W\left(t_{1}-t_{2}\right)= \\
=2 \gamma m \int\left(\frac{1-e^{-\beta \lambda}}{\lambda}\right)^{-1} \frac{1}{\gamma^{2}+\lambda^{2}} e^{-i \lambda\left(t_{1}-t_{2}\right)} \frac{d \lambda}{2 \pi}
\end{gathered}
$$

Заметим, что преобразование Фурье двухточечной функции $-m^{2} \partial_{t}^{2} W(t)$ импульсного поля является мультипликатором в пространстве Шварца $\mathcal{S}(\mathbb{R})$. Соответственно $-m^{2} \partial_{t}^{2} W(t)$ есть свертыватель в $\mathcal{S}(\mathbb{R})$. Отсюда следует, что для любых $g_{1}, g_{2} \in \mathcal{S}_{\mathrm{r}}(\mathbb{R})$ среднее

$$
\left\langle p_{g_{1}}(t) p_{g_{2}}\right\rangle \rightarrow 0 \quad \text { при } \quad t \rightarrow \infty .
$$

Здесь и далее $p_{g}(t) \equiv \int p(t+\tau) g(\tau) d \tau, g \in \mathcal{S}_{\mathrm{r}}(\mathbb{R})$. Это замечание позволяет вывести важное свойство сильного перемешивания гиббсовского состояния $s^{(0)}$. Именно, для любого состояния импульсного поля с матрицей плотности $\rho^{(0)}$ в $\mathcal{H}^{(0)}$ имеет место предельное соотношение (где $f=-\partial_{t} g, g \in \mathcal{S}_{\mathrm{r}}(\mathbb{R})$ )

$$
\operatorname{tr}\left(\rho^{(0)} e^{(i / m) p_{g}^{(0)}(t)}\right) \rightarrow s^{(0)}\left(\mathcal{E}_{f}\right) \quad \text { при } \quad t \rightarrow \infty .
$$


Для доказательства (40) используем понятие температурной нормальной экспоненты импульсного поля (где по-прежнему $f=-\partial_{t} g, g \in \mathcal{S}_{\mathrm{r}}(\mathbb{R})$ )

$$
N E_{f}^{(0)} \equiv N e^{(i / m) p_{g}^{(0)}}=E_{f}^{(0)}\left\langle\mathcal{E}_{f}\right\rangle^{-1}
$$

Это выражение можно рассматривать как производящий функционал температурных нормальных произведений $N\left(p^{(0)}\left(t_{1}\right) \ldots p^{0}\left(t_{n}\right)\right)$, симметричных по индексам $1,2, \ldots, n$. (Отметим, что на некоторой плоской области векторов эти произведения являются операторными обобщенными функциями по $t_{1}$, гладко зависящими от $t_{1}-t_{1}, \ldots, t_{n}-t_{1}$.) Нетрудно проверить, что поля под знаком $N$-произведения коммутируют,

$$
\begin{gathered}
\left\langle\mathrm{GIBBS}\left|N E_{f}^{(0)}\right| \mathrm{GIBBS}\right\rangle_{0}=1, \\
N E_{f_{1}}^{(0)} N E_{f_{2}}^{(0)}=\exp \left(-\frac{1}{m^{2}}\left\langle p_{g_{1}} p_{g_{2}}\right\rangle\right) N E_{f_{1}+f_{2}}^{(0)},
\end{gathered}
$$

где $f_{j}=-\partial_{t} g_{j}$. Из соотношений $(39),(42),(43)$ легко получить, что при $t \rightarrow \infty$

$$
\left\langle\operatorname{GIBBS}\left|N E_{f_{1}}^{(0)} N \exp \left(\frac{i}{m} p_{g}^{(0)}(t)\right) N E_{f_{2}}^{(0)}\right| \mathrm{GIBBS}\right\rangle_{0} \rightarrow\left\langle\mathrm{GIBBS}\left|N E_{f_{1}}^{(0)} N E_{f_{2}}^{(0)}\right| \mathrm{GIBBS}\right\rangle_{0} .
$$

В силу цикличности представления $\pi^{(0)}$ с циклическим вектором $\mid$ GIBBS $\rangle$ любая матрица плотности в $\mathcal{H}^{(0)}$ может быть приближена с произвольной точностью по tr-норме линейными комбинациями операторов конечного ранга вида

$$
\sigma=N E_{f_{2}}^{(0)}|\mathrm{GIBBS}\rangle\langle\mathrm{GIBBS}| N E_{f_{1}}^{(0)},
$$

где $f_{j}=-\partial_{t} g_{j}$. Следовательно, из (44) следует (40).

3.3. Конструкция температурного представления алгебры $\mathcal{A}$. Обратимся к конструкции температурного представления $\pi$ для всей алгебры $\mathrm{KKC} \mathcal{A}$. Определим равновесное обобщенное состояние $\mathfrak{s}$ (с температурой $T$ ) характеристическим функционалом

$$
\begin{aligned}
\mathfrak{s}\left(\mathcal{E}_{f}\right) & =2 \pi \delta(\tilde{f}(0)) e^{(-1 / 2)\left\langle\left(q_{f}\right)^{2}\right\rangle}= \\
& =2 \pi \delta(\tilde{f}(0)) \exp \left(-\frac{1}{2} \int W\left(t_{1}-t_{2}\right) f\left(t_{1}\right) f\left(t_{2}\right) d t_{1} d t_{2}\right), \quad f \in \mathcal{S}_{\mathrm{r}}(\mathbb{R}) .
\end{aligned}
$$

Этот функционал назван обобшенным состоянием, потому что он ненормируем (его норма $\left.\mathfrak{s}(1)=\mathfrak{s}\left(\mathcal{E}_{0}\right)=\infty\right)$. Для получения числовых значений функционала $\mathfrak{s}$ нужно в $(46)$ заменить $\mathcal{E}_{f}$ на сглаженные элементы $A$ согласно $(29)$, где меры $d \mu(f)$ должны быть $\tilde{f}(0)$-гладкими. На сглаженных таким образом элементах $A$ абстрактной алгебры КKС условие положительности $\mathfrak{s}\left(A^{*} A\right) \geqslant 0$ выполняется, и по аналогии с конструкцией ГНС 
мы получаем температурное представление $\pi$ алгебры $\mathrm{KKC} \mathcal{A}$ в некотором гильбертовом пространстве $\mathcal{H}$ (вместо циклического вектора теперь появляется циклический обобщенньй вектор $|\mathrm{EQUI}\rangle$ с нормой $\infty)$.

Гильбертово пространство $\mathcal{H}$ порождается обобшенными векторами $Y\left(\mathcal{E}_{f}\right)$, $f \in \mathcal{S}_{\mathrm{r}}(\mathbb{R})$, со скалярными произведениями ${ }^{3)}$

$$
\left\langle Y\left(\mathcal{E}_{f_{1}}\right) \mid Y\left(\mathcal{E}_{f_{2}}\right)\right\rangle=2 \pi \delta\left(\tilde{f}_{1}(0)-\tilde{f}_{2}(0)\right) s^{(0)}\left(\mathcal{E}_{f_{1}}^{*} \mathcal{E}_{f_{2}}\right), \quad f_{1}, f_{2} \in \mathcal{S}_{\mathrm{r}}(\mathbb{R}) .
$$

После сглаживания $Y\left(\mathcal{E}_{f}\right)$ с $\tilde{f}(0)$-гладкой мерой $d \mu(f)$ по $f$, так же как и в формуле $(29)$, получается обычный вектор $Y(A) \in \mathcal{H}$. Соответственно, сглаживая (47) по $f_{1}, f_{2}$, мы получаем числовое значение скалярного произведения $\left\langle Y\left(A_{1}\right) \mid Y\left(A_{2}\right)\right\rangle$. Строго говоря, отображение $Y: A \rightarrow Y(A)$ есть линейное отображение подалгебры (без единицы) элементов $A$ вида $(29)$ с $\tilde{f}(0)$-гладкими мерами $d \mu(f)$ в гильбертово пространство $\mathcal{H}$; эти элементы образуют плотное в $\mathcal{H}$ комплексное линейное подпространство. Операторы $\pi\left(\mathcal{E}_{f}\right)=E_{f}=e^{i q_{f}}$ представления $\pi$ определяются формулой

$$
\pi\left(\mathcal{E}_{f}\right) Y\left(\mathcal{E}_{f_{1}}\right)=Y\left(\mathcal{E}_{f} \mathcal{E}_{f_{1}}\right)
$$

Разумеется, это соотношение также подлежит сглаживанию по $f_{1}$ с $\tilde{f}(0)$-гладкой мерой $d \mu\left(f_{1}\right)$. В результате получается векторная формула в обычном (а не в обобщенном) смысле:

$$
\pi\left(\mathcal{E}_{f}\right) Y(A)=Y\left(\mathcal{E}_{f} A\right)
$$

Грубо говоря, обобшенные векторы $Y\left(\mathcal{E}_{f}\right)$ получаются действием $\pi\left(\mathcal{E}_{f}\right)$ на упомянутый циклический обобшенный вектор $|\mathrm{EQUI}\rangle$, однако мы не будем заниматься уточнением этой формулировки.

Обобшенное состояние $\mathfrak{s}$ выше было названо равновесным вследствие его инвариантности относительно сдвигов по времени $(17)$, при которых $\mathcal{E}_{f} \rightarrow \mathcal{E}_{f_{\tau}}$, где $f_{\tau}(t)=f(t+\tau)$, $\tau \in \mathbb{R}$. Следовательно, преобразования динамической групшы реализуются унитарными операторами $U(\tau)=e^{-i H \tau}$ по формуле $U(\tau) Y\left(\mathcal{E}_{f}\right)=Y\left(\mathcal{E}_{f_{\tau}}\right)$ или, после сглаживания по $f, U(\tau) Y(A)=Y\left(A_{\tau}\right)$. Оператор $H$ есть гамильтониан (энергия) в представлении $\pi$ (циклический обобшенный вектор $|\mathrm{EQUI}\rangle$ инвариантен относительно $U(\tau)$, и его энергия принимается за нулевой уровень). Явное выражение для гамильтониана $H$ получается из (18) приписыванием знака температурного нормального произведения

$$
H=\frac{1}{2} \int N\left((\xi(t))^{2}\right) d t
$$

Температурные нормальные произведения полей в $\mathcal{H}$ определяются формулой типа (41), т.е. заменой $E_{f}^{(0)}, p^{(0)}(t)$ на $E_{f}, p(t)$ (в формуле (50) следует иметь в виду, что функция $\xi(t) \equiv \partial_{t} \varphi^{\text {in }}(t)$ выражается через $p(t)$ согласно уравнению Ланжевена).

\footnotetext{
${ }^{3)}$ В формулах $(51),(52)$ работы [10] вместо $\mathcal{E}_{-f_{1}+f_{2}}$ и $\mathcal{E}_{-f_{1}+f+f_{2}}$ должно быть $\mathcal{E}_{-f_{1}} \mathcal{E}_{f_{2}}$ и $\mathcal{E}_{-f_{1}} \mathcal{E}_{f} \mathcal{E}_{f_{2}}$
} 


\section{4. РАЗЛОЖЕНИЕ ПО ПОЛНОМУ ИМПУЛЬСУ}

4.1. Оператор полного импульса. Данный раздел является этапом в определении коллективной координаты (см. раздел 1). Полученные формулы позволят показать в следующем разделе, что каждое температурное представление $\pi$ алгебры $\mathrm{KKC} \mathcal{A}$ унитарно эквивалентно соответствуюшему представлению, построенному методом выделения коллективной координаты, что делает более наглядной структуру представления $\pi$.

Обобщенное состояние $\mathfrak{s}$ инвариантно относительно пространственных трансляций (22), (28), которые вследствие этого реализуются унитарными операторами $V(r)=e^{i K r}$, действующими по формуле

$$
V(r) Y\left(\mathcal{E}_{f}\right)=Y\left(\Gamma_{r}\left(\mathcal{E}_{f}\right)\right)=e^{i \tilde{f}(0) r} Y\left(\mathcal{E}_{f}\right),
$$

где $r \in \mathbb{R}, f \in \mathcal{S}_{\mathrm{r}}(\mathbb{R})$, или после сглаживания по $f$

$$
V(r) Y(A)=Y\left(\Gamma_{r}(A)\right)
$$

Оператор $K$ задает полный импульс системы “частица+термостат” в представлении $\pi$,

$$
K Y\left(\mathcal{E}_{f}\right)=\tilde{f}(0) Y\left(\mathcal{E}_{f}\right)
$$

Нетрудно видеть, что представления $U(\tau)$ и $V(\tau)$ коммутируют, следовательно, коммутируют их генераторы $[H, K]=0$. Из формулы

$$
V(r) E_{f} V(r)^{-1}=e^{i \tilde{f}(0) r} E_{f}
$$

или из формулы

$$
e^{i K r} e^{i q_{f}} e^{-i K r}=e^{i\left(q_{f}+r \tilde{f}(0)\right)}
$$

следует коммутационное соотношение между оператором $K$ и координатным полем,

$$
[q(t), K]=i
$$

а также соотношение

$$
[p(t), K]=0 .
$$

Оператор $K$ выражается интегралом типа (24) от импульсного поля. В квантовом случае это равенство следует понимать в смысле следуюшего предела в слабой операторной топологии:

$$
e^{(i r \gamma / 2) p_{u_{n}}} \rightarrow e^{i K r}, \quad n \rightarrow \infty
$$

где $r \in \mathbb{R}, \quad u_{n}(t)$ - последовательность функций из $\mathcal{S}_{\mathrm{r}}(\mathbb{R})$, обладающих свойством $u_{n}(t) \rightarrow 1$ в $\mathcal{S}^{\prime}(\mathbb{R})$ и $\left\langle\mathrm{GIBBS}\left|\left(p_{u_{n}}\right)^{2}\right| \mathrm{GIBBS}\right\rangle \rightarrow 0$ при $n \rightarrow \infty$ (доказательство аналогично выводу формулы (11.49) в работе [12]). Отсюда вытекает, что оператор полного импульса присоединен к алгебре фон Неймана импульсного поля в представлении $\pi$. 
4.2. Обобщенные собственные подпространства полного импульса. Гильбертово пространство $\mathcal{H}$ представления $\pi$ разлагается в прямой интеграл

$$
\int^{\oplus} \mathcal{H}^{(k)} \frac{d k}{2 \pi}
$$

обобщенных подпространств $\mathcal{H}^{(k)}$ в $\mathcal{H}$ с определенными значениями $k$ оператора $K$. При любом $k \in \mathbb{R}$ обозначим посредством $\mathcal{A}^{(k)}$ комплексное линейное подпространство в абстрактной алгебре $\mathrm{KKC} \mathcal{A}$, порожденное элементами $\mathcal{E}_{f}$ при $f \in \mathcal{F}^{(k)}$ (см. формулу (1)). Тогда по аналогии с конструкцией ГНС для представления $\pi^{(0)}$ в $\mathcal{H}^{(0)}$ можно утверждать, что сушествуют гильбертово пространство $\mathcal{H}^{(k)}$ и линейный оператор

$$
\mathcal{A}^{(k)} \ni A \rightarrow Y^{(k)}(A) \in \mathcal{H}^{(k)}
$$

из $\mathcal{A}^{(k)}$ на плотное линейное подпространство в $\mathcal{H}^{(k)}$ такие, что для скалярного произведения в $\mathcal{H}^{(k)}$ выполняются определяюшие соотношения

$$
\left\langle Y^{(k)}\left(\mathcal{E}_{f_{1}}\right) \mid Y^{(k)}\left(\mathcal{E}_{f_{2}}\right)\right\rangle_{k}=\mathfrak{s}^{(0)}\left(\mathcal{E}_{f_{1}}^{*} \mathcal{E}_{f_{2}}\right) \equiv\left\langle\mathrm{GIBBS}\left|\pi^{(0)}\left(\mathcal{E}_{f_{1}}^{*} \mathcal{E}_{f_{2}}\right)\right| \mathrm{GIBBS}\right\rangle_{0},
$$

где $f_{1}, f_{2} \in \mathcal{F}^{(k)}$.

В связи с разложением (55) мы вводим аналоги унитарных операторов $\pi\left(\mathcal{E}_{f}\right)$. А именно, при любых $k, k_{1} \in \mathbb{R}, f \in \mathcal{F}^{(k)}$ мы определяем изометрические операторы $\pi^{\left(k_{1}+k \leftarrow k_{1}\right)}\left(\mathcal{E}_{f}\right)$ из $\mathcal{H}^{\left(k_{1}\right)}$ на $\mathcal{H}^{\left(k_{1}+k\right)}$ по формуле

$$
\pi^{\left(k_{1}+k \leftarrow k_{1}\right)}\left(\mathcal{E}_{f}\right) Y^{\left(k_{1}\right)}\left(\mathcal{E}_{f_{1}}\right)=Y^{\left(k_{1}+k\right)}\left(\mathcal{E}_{f} \mathcal{E}_{f_{1}}\right)
$$

при $f_{1} \in \mathcal{F}^{\left(k_{1}\right)}$. Очевидно, выполняется цепное правило

$$
\pi^{\left(k+k_{1}+k_{2} \leftarrow k\right)}\left(\mathcal{E}_{f_{1}} \mathcal{E}_{f_{2}}\right)=\pi^{\left(k+k_{1}+k_{2} \leftarrow k+k_{2}\right)}\left(\mathcal{E}_{f_{1}}\right) \pi^{\left(k+k_{2} \leftarrow k\right)}\left(\mathcal{E}_{f_{2}}\right)
$$

при любых $f_{j} \in \mathcal{F}^{\left(k_{j}\right)}$. В частности, действуя на вектор $|\mathrm{GIBBS}\rangle \equiv Y^{(0)}\left(\mathcal{E}_{0}\right)$ из $\mathcal{H}^{(0)}$, операторы $\pi^{(k \leftarrow 0)}\left(\mathcal{E}_{f}\right)$ при $f \in \mathcal{F}^{(k)}$ переводят его в векторы

$$
Y^{(k)}\left(\mathcal{E}_{f}\right)=\pi^{(k \leftarrow 0)}\left(\mathcal{E}_{f}\right)|\mathrm{GIBBS}\rangle,
$$

образуюшие тотальное множество в $\mathcal{H}^{(k)}$ (это означает, что комплексные линейные комбинации этих векторов образуют плотное линейное подпространство в $\left.\mathcal{H}^{(k)}\right)$.

Прямой интеграл (55) можно считать пополнением предгильбертова пространства непрерывных функций

$$
\Psi: \mathbb{R} \ni k \rightarrow \Psi(k) \in \mathcal{H}^{(k)}
$$

с компактными носителями. Здесь понятие непрерывности нуждается в уточнении (учитываюшем, что $\Psi$ есть, в сущности, сечение расслоения $\bigcup_{k} \mathcal{H}^{(k)}$ гильбертовых пространств). А именно, функция (61) считается непрерывной, если функция

$$
\mathbb{R} \ni k \rightarrow \pi^{(0 \leftarrow k)}\left(\mathcal{E}_{-k u}\right) \Psi(k) \in \mathcal{H}^{(0)}
$$

5 Теоретическая и математическая физика, т. 136, № 1, 2003 г. 
из $\mathbb{R}$ в $\mathcal{H}^{(0)}$ непрерывна в сильной топологии $\mathcal{H}^{(0)}$, где $u$ - любая фиксированная функция из $\mathcal{F}^{(1)}$.

Скалярное произведение функций типа (61) дается формулой

$$
\left\langle\Psi_{1} \mid \Psi_{2}\right\rangle=\int\left\langle\Psi_{1}(k) \mid \Psi_{2}(k)\right\rangle_{k} \frac{d k}{2 \pi} .
$$

По сути дела, функция (62) есть элемент тензорного произведения $\mathcal{L}^{2}(\mathbb{R}, d k / 2 \pi) \otimes \mathcal{H}^{(0)}$. Для дальнейшего заметим, что некоторое множество функций вида (61) плотно в (55), если множество соответствующих функций $(62)$ плотно в $\mathcal{L}^{2}(\mathbb{R}, d k / 2 \pi) \otimes \mathcal{H}^{(0)}$. Под разложением $\mathcal{H}$ в прямой интеграл (55) мы подразумеваем теперь изометрический оператор

$$
\mathcal{V}: \mathcal{H} \rightarrow \int^{\oplus} \mathcal{H}^{(k)} \frac{d k}{2 \pi}
$$

из $\mathcal{H}$ на все пространство (55). Этот оператор сопоставляет вектору $Y(A)$ для $A$ вида $(29)$ с $\tilde{f}(0)$-гладкой мерой $d \mu(f)$ вектор $\Psi$ вида $(61)$, где $\Psi(k) \in \mathcal{H}^{(k)}$ определяется скалярными произведениями

$$
\left\langle Y^{(k)}(B) \mid \Psi(k)\right\rangle=2 \pi \int_{\mathcal{M}}\left\langle Y^{(k)}(B) \mid Y^{(k)}\left(\mathcal{E}_{f}\right)\right\rangle \delta(k-\tilde{f}(0)) d \mu(f)
$$

при любых $B \in \mathcal{A}^{(k)}$. Это выражение как антилинейный функционал на плотном в $\mathcal{H}^{(k)}$ линейном подпространстве векторов $Y^{(k)}(B)$ непрерывен с нормой, не превосходяшей

$$
\sum_{i=1}^{n} \int_{\mathcal{M}_{i}} 2 \pi \delta(k-\tilde{f}(0))\left|F_{i}(f)\right| d l_{i}(f)
$$

(см. представление (30)). Следовательно, формула (65) действительно определяет вектор $\Psi(k) \in \mathcal{H}^{(k)}$.

Нетрудно проверить далее, что если векторы $Y\left(A_{i}\right)$ имеют вид $(29)$ с $\tilde{f}(0)$-гладкими мерами $d \mu_{1}(f), d \mu_{2}(f)$, то для соответствуюших векторов $\Psi_{1}, \Psi_{2}$ выполняется соотношение

$$
\left\langle\Psi_{1} \mid \Psi_{2}\right\rangle=\left\langle Y\left(A_{1}\right) \mid Y\left(A_{2}\right)\right\rangle .
$$

Следовательно, изометрический оператор (64) корректно определен. В частности, согласно (65) векторы $Y^{(k)}(A)$ для $A$ вида

$$
A=\int F(k) \mathcal{E}_{\left(k-k_{1}\right) u} \mathcal{E}_{f} \frac{d k}{2 \pi}
$$

при $u \in \mathcal{F}^{1}, f \in \mathcal{F}^{\left(k_{1}\right)}, F(k) \in \mathcal{S}(\mathbb{R})$ по $k$ переводятся оператором $\mathcal{V}$ в векторы из (55) вида

$$
\Psi(k)=F(k) Y^{(k)}\left(\mathcal{E}_{\left(k-k_{1}\right) u} \mathcal{E}_{f}\right) .
$$

Так как векторы $Y^{(0)}\left(\mathcal{E}_{-k_{1} u} \mathcal{E}_{f}\right)$ образуют тотальное множество в $\mathcal{H}^{(0)}$, то векторы $F(k) \otimes Y^{(0)}\left(\mathcal{E}_{-k_{1} u} \mathcal{E}_{f}\right)$ образуют тотальное множество векторов в $\mathcal{L}^{2}(\mathbb{R}, d k / 2 \pi) \otimes \mathcal{H}^{(0)}$. Согласно сделанному выше замечанию в связи с формулами (61), (62) последнее означает, что векторы (67) образуют тотальное множество в (55). Это доказывает, что $\mathcal{V}$ есть изоморфизм. 
4.3. Реализация температурного представления посредством подпространств полного импульса. Оператору $E_{f}$ c $f \in \mathcal{F}^{\left(k_{1}\right)}$, действующему в $\mathcal{H}$, соответствует оператор $\mathcal{V} E_{f} \mathcal{V}^{-1}$, действующий в (55) по формуле

$$
\left(\mathcal{V} E_{f} \mathcal{V}^{-1} \Psi\right)(k)=\pi^{\left(k \leftarrow k-k_{1}\right)}\left(\mathcal{E}_{f}\right) \Psi\left(k-k_{1}\right)
$$

В частности, пусть $f \in \mathcal{F}^{(0)}, f=-\partial_{t} g$ при $g \in \mathcal{S}_{\mathrm{r}}(\mathbb{R})$. Тогда операторы $\pi^{(k \leftarrow k)}\left(\mathcal{E}_{f}\right)$ являются унитарными операторами в $\mathcal{H}^{(k)}$, задавая тем самым представление $\pi^{(k)}$ алгебры $\mathcal{A}^{(0)}$ и, следовательно, представление $p^{(k)}(t)$ импульсного поля в $\mathcal{H}^{(k)}$ :

$$
\pi^{(k)}\left(\mathcal{E}_{f}\right) \equiv \pi^{(k \leftarrow k)}\left(\mathcal{E}_{f}\right)=\exp \left(\frac{i}{m} \int p^{(k)}(t) g(t) d t\right) .
$$

Вместе с формулой (68) это означает, что импульсное поле $p(t)$ приводится несобственными (относительно $\mathcal{H}$ ) подпространствами $\mathcal{H}^{(k)}$, в результате чего представление подалгебры $\mathcal{A}^{(0)} \mathrm{KKC} \mathrm{в}(55)$ разлагается в прямой интеграл представлений

$$
\left.\mathcal{V}\right|_{\mathcal{A}^{(0)}} \mathcal{V}^{-1}=\int^{\oplus} \pi^{(k)} \frac{d k}{2 \pi}
$$

Очевидно, оператору полного импульса $K$ соответствует оператор $\mathcal{V} K \mathcal{V}^{-1}$, действующий в (55) как оператор умножения,

$$
\left(\mathcal{V} K \mathcal{V}^{-1} \Psi\right)(k)=k \Psi(k)
$$

Из (54) следует также, что для всех $r \in \mathbb{R}$

$$
e^{i r(\gamma / 2) p_{u n}^{(k)}} \rightarrow e^{i k r} \quad \text { при } \quad n \rightarrow \infty .
$$

Как и для вакуумного представления ( $T=0$, см. [12], гл. 11), в рассматриваемом случае $(T>0)$ представления $\pi^{(k)}$ алгебры $\mathcal{A}^{(0)}$ в $\mathcal{H}^{(k)}$ попарно унитарно неэквивалентны. Действительно, предположение о сушествовании изометрического оператора $\mathcal{U}$, обладающего свойством $\mathcal{U} \pi^{(k)}(A) \mathcal{U}^{-1}=\pi^{\left(k_{1}\right)}(A)$ при всех $A \in \mathcal{A}^{(0)}$, для некоторых $k \neq k_{1}$ противоречило бы соотношению (72).

Представления $\pi^{(k)}$ импульсного поля получаются из $\pi^{(0)}$ линейными неоднородными каноническими преобразованиями

$$
p^{(0)}(t) \rightarrow \nu(t) k+p^{(0)}(t), \quad \text { где } \quad \frac{\gamma}{2} \nu(t) \in \mathcal{F}^{(1)} .
$$

Докажем это. Положим

$$
\begin{aligned}
w(t) & =\frac{1}{2 \gamma}\left(\gamma^{2}-\partial_{t}^{2}\right) \nu(t) \in \mathcal{F}^{(1)}, \\
U^{(k)} & =\pi^{(0 \leftarrow k)}\left(\mathcal{E}_{-k w}\right) .
\end{aligned}
$$


Тогда для всех $g \in \mathcal{S}_{\mathrm{r}}(\mathbb{R}), f(t)=-\partial_{t} g(t)$ имеем

$$
\mathcal{U}^{(k)} e^{(i / m) p_{g}^{(k)}}\left(\mathcal{U}^{(k)}\right)^{-1}=\pi^{(0 \leftarrow k)}\left(\mathcal{E}_{-k w}\right) \pi^{(k)}\left(\mathcal{E}_{f}\right) \pi^{(k \leftarrow 0)}\left(\mathcal{E}_{-k w}\right)=\pi^{(0)}\left(\mathcal{E}_{-k w} \mathcal{E}_{f} \mathcal{E}_{k w}\right) .
$$

С помошью КKC (27) это выражение приводится к виду

$$
\begin{aligned}
& e^{(i / m) p_{g}^{(0)}} e^{(-k / m)\left[p_{g}, q_{w}\right]}= \\
& \quad=e^{(i / m) p_{g}^{(0)}} \exp \left(\frac{i k}{m} \int e^{-\gamma\left|t_{1}-t_{2}\right|} g\left(t_{1}\right) \frac{1}{2 \gamma}\left(\gamma^{2}-\partial_{t_{2}}^{2}\right) \nu\left(t_{2}\right) d t_{1} d t_{2}\right) .
\end{aligned}
$$

Отсюда получаем

$$
p^{(k)}(t)=\left(\mathcal{U}^{(k)}\right)^{-1}\left(p^{(0)}(t)+\nu(t) k\right) \mathcal{U}^{(k)},
$$

что доказывает унитарную эквивалентность $\pi^{(k)}$ представлению $p^{(0)}(t)+\nu(t) k$ импульсного поля в $\mathcal{H}^{(0)}$. Стало быть, представления $\pi^{(k)}$ при $k \neq 0$ можно назвать смешенными гиббсовскими представлениями импульсного поля - по аналогии со случаем $T=0$ (см. [12], гл. 11), где $\pi^{(k)}$ при $k \neq 0$ именовались смешенными фоковскими представлениями. Канонические преобразования (73) при $k \neq 0$ являются несобственными (т.е. унитарно нереализуемыми). Это означает, что не существует унитарного оператора $\mathcal{U}$ в $\mathcal{H}^{(0)}$ такого, что $\mathcal{U} p^{(0)}(t) \mathcal{U}^{-1}=p^{(0)}(t)+\nu(t) k$ (существование $\mathcal{U}$ противоречило бы свойству (72)).

\section{5. КОЛЛЕКТИВНЫЕ И ФОНОННЫЕ СОСТАВЛЯЮЩИЕ}

5.1. Упрощение структуры пространства температурного представления.

В этом разделе мы упростим структуру гильбертова пространства $\mathcal{H}$ и каждого температурного представления $\pi$. Для этого используем сведение представлений $\pi^{(k)}$ импульсного поля к гиббсовскому представлению $\pi^{(0)}$ (в пространстве $\mathcal{H}^{(0)}$ ) каноническими преобразованиями (73), (79) и реализуем температурное представление в тензорном произведении

$$
\underset{\sim}{\mathcal{H}}=\mathcal{L}^{2}\left(\mathbb{R}, \frac{d k}{2 \pi}\right) \otimes \mathcal{H}^{(0)}
$$

При этом пространство $\underset{\sim}{\mathcal{H}}$ рассматривается как пополнение предгильбертова пространства непрерьвных функций (в сильной топологии $\mathcal{H}^{(0)}$ )

$$
\Phi: \mathbb{R} \ni k \rightarrow \Phi(k) \in \mathcal{H}^{(0)}
$$

из $\mathbb{R}$ в $\mathcal{H}^{(0)}$ с компактными носителями и со скалярными произведениями

$$
\left\langle\Phi_{1} \mid \Phi_{2}\right\rangle=\int\left\langle\Phi_{1}(k) \mid \Phi_{2}(k)\right\rangle_{0} \frac{d k}{2 \pi} .
$$

Действием на первый тензорньй сомножитель в (77) зададим эрмитовы операторы $K$, $X=i \partial_{k}$ и унитарные операторы $e^{i k_{1} X}$ при $k_{1} \in \mathbb{R}$ в пространстве $\underset{\sim}{\mathcal{H}}:$

$$
(K \Phi)(k)=k \Phi(k), \quad(X \Phi)(k)=i \partial_{k} \Phi(k), \quad\left(e^{i k_{1} X} \Phi\right)(k)=\Phi\left(k-k_{1}\right),
$$


так что

$$
[X, K]=i
$$

Кроме того, перенесем действие полей $p^{(0)}(t)$ и $\xi^{(0)}(t)$, заданных в $\mathcal{H}^{(0)}$, на все пространство $\underset{\sim}{\mathcal{H}}$, считая, что эти поля действуют на второй тензорный сомножитель в $(77)$, т.е. полагая

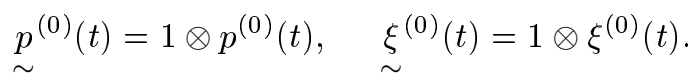

Для дальнейшего удобно отождествить пространства $\mathcal{H}$ и $(55)$, так что теперь мы считаем, что в формуле $(64) \mathcal{V}=1$. Фиксируем функцию $\nu(t) \in \mathcal{S}_{\mathrm{r}}(\mathbb{R})$ такую, что $\gamma \nu(t) / 2 \in \mathcal{F}^{(1)}$ и определим $w(t) \in \mathcal{F}^{(1)}$ формулой $(74)$. Функцию $\chi(t)$ класса $\varepsilon * \mathcal{S}_{\mathrm{r}}(\mathbb{R})$ зададим формулой

$$
\chi(t)=\frac{1}{2 m} \varepsilon(t) * w(t) .
$$

Кроме того, определим функцию $\eta(t) \in \mathcal{S}_{\mathrm{r}}(\mathbb{R})$ уравнением ланжевеновского типа

$$
\left(\gamma+\partial_{t}\right) \nu(t)=\sqrt{2 \gamma m} \eta(t)
$$

Изометрический оператор

$$
\mathcal{W}: \mathcal{H}=\int^{\oplus} \mathcal{H}^{(k)} \frac{d k}{2 \pi} \rightarrow \underset{\sim}{\mathcal{H}}=\mathcal{L}^{2}\left(\mathbb{R}, \frac{d k}{2 \pi}\right) \otimes \mathcal{H}^{(0)}
$$

из $(55)$ в (77) зададим формулой $\mathcal{W}: \Psi \rightarrow \Phi$, где

$$
(\mathcal{W} \Psi)(k)=\Phi(k)=\pi^{(0 \leftarrow k)}\left(\mathcal{E}_{-k w}\right) \Psi(k)
$$

В результате мы имеем новое представление $\mathrm{KKC}_{\text {в }}^{\mathcal{H}} \underset{\sim}{\mathcal{H}}$, унитарно эквивалентное прежнему температурному представлению $\pi$. Мы будем снабжать нижней тильдой операторы в новом представлении,

$$
\underset{\sim}{E_{f}}=\mathcal{W} E_{f} \mathcal{W}^{-1}=e^{i q_{f}}, \quad f \in \mathcal{S}_{\mathrm{r}}(\mathbb{R})
$$

Соответственно через $\underset{\sim}{q}(t), \underset{\sim}{p}(t), \sqrt{2 \gamma m} \underset{\sim}{\xi}(t)$ обозначим координатное, импульсное поля и флуктуируюшую силу в новом представлении,

$$
\underset{\sim}{q}(t)=\mathcal{W} q(t) \mathcal{W}^{-1}, \quad \underset{\sim}{p}(t)=\mathcal{W} p(t) \mathcal{W}^{-1}, \quad \underset{\sim}{\xi}(t)=\mathcal{W} \xi(t) \mathcal{W}^{-1}
$$

Эти величины по-прежнему удовлетворяют уравнению Ланжевена (2). Для оператора полного импульса $\mathcal{W} K \mathcal{W}^{-1}$ сохраним прежнее обозначение $K$. 
5.2. Определение коллективных и фононных составляющих. Основной результат данного раздела состоит в том, что операторы (88) нового представления разлагаются в суммы операторов, которые мы назовем соответственно коллективными и фононными составляюшими,

$$
\underset{\sim}{q(t)}=q^{\mathrm{col}}(t)+{\underset{\sim}{q}}^{(0)}(t), \quad \underset{\sim}{p}(t)=p^{\mathrm{col}}(t)+{\underset{\sim}{p}}^{(0)}(t), \quad \underset{\sim}{\xi}(t)=\xi^{\mathrm{col}}(t)+{\underset{\sim}{\xi^{(0)}}(t) .}^{(}
$$

Коллективные составляюшие $q^{\mathrm{col}}(t), p^{\mathrm{col}}(t), \xi^{\mathrm{col}}(t)$ действуют на первый тензорный сомножитель в (77) как

$$
q^{\mathrm{col}}(t)=\chi(t) K+X, \quad p^{\mathrm{col}}(t)=\nu(t) K, \quad \xi^{\mathrm{col}}(t)=\eta(t) K .
$$

В силу (81) при преобразованиях пространственных трансляций составляюшая $q^{\mathrm{col}}(t)$ преобразуется по правилу

$$
e^{i K r} q^{\mathrm{col}}(t) e^{-i K r}=q^{\mathrm{col}}(t)+r
$$

т.е. как оператор координаты в формуле (22). Поэтому мы назовем $q^{\mathrm{col}}(t)$ коллективной координатой для данной симметрии группы $\mathbb{R}$.

Фононные составляюшие определены формулами (82) и формулой

$$
{\underset{\sim}{q}}^{(0)}(t)=\frac{1}{2 m} \int{\underset{\sim}{p}}^{(0)}(\tau)(\varepsilon(t-\tau)+\varepsilon(\tau) * w(\tau)) d \tau .
$$

Результат сглаживания $\underset{\sim}{q(0)}(t)$ с основной функцией $f(t) \in \mathcal{S}_{\mathrm{r}}(\mathbb{R})$ дает

$$
\int \underset{\sim}{q^{(0)}}(t) f(t) d t=-\frac{1}{2 m} \int{\underset{\sim}{p}}^{(0)}(t) \varepsilon(t) *(f(t)-\tilde{f}(0) w(t)) d t,
$$

где $(f(t)-\tilde{f}(0) w(t)) \in \mathcal{F}^{(0)}$, так что $\varepsilon(t) *(f(t)-\tilde{f}(0) w(t)) \in \mathcal{S}_{\mathrm{r}}(\mathbb{R})$. Фононные составляющие дополняют коллективную координату до полного набора динамических переменных. Они действуют на второй тензорный сомножитель в (77) и коммутируют с оператором $K$, т.е. они являются инвариантами указанной симметрии группы $\mathbb{R}$.

Из данных определений следуют очевидные соотношения:

$$
p^{\mathrm{col}}(t)=m \partial_{t} q^{\mathrm{col}}(t), \quad \underset{\sim}{p^{(0)}}(t)=m \partial_{t}{\underset{\sim}{(0)}}^{(t)} .
$$

Уравнение Ланжевена (2) распадается на два отдельных уравнения для каждого типа составляющих,

$$
\begin{aligned}
m\left(\gamma+\partial_{t}\right) \partial_{t} q^{\mathrm{col}}(t) & =\sqrt{2 \gamma m} \xi^{\mathrm{col}}(t), \\
m\left(\gamma+\partial_{t}\right) \partial_{t} q^{(0)}(t) & =\sqrt{2 \gamma m} \underset{\sim}{\xi^{(0)}}(t),
\end{aligned}
$$

причем переменные первого уравнения коммутируют с переменными второго уравнения. 
5.3. Доказательство формул (89). Пусть $\Psi \in \mathcal{H}, \Phi=\mathcal{W} \Psi \in \mathcal{H}$, тогда

$$
\begin{aligned}
\underset{\sim}{p(t) \Phi)(k)} & =\left(\mathcal{W} p(t) \mathcal{W}^{-1} \Phi\right)(k)=(\mathcal{W} p(t) \Psi)(k)= \\
& =\pi^{(0 \leftarrow k)}\left(\mathcal{E}_{-k w}\right)(p(t) \Psi)(k)=\pi^{(0 \leftarrow k)}\left(\mathcal{E}_{-k w}\right) p^{(k)}(t) \Psi(k) .
\end{aligned}
$$

Учитывая соотношения (75), (76), имеем

$$
\begin{aligned}
\underset{\sim}{p(t) \Phi)(k)} & =\pi^{(0 \leftarrow k)}\left(\mathcal{E}_{-k w}\right) p^{(k)}(t) \pi^{(k \leftarrow 0)}\left(\mathcal{E}_{-k w}\right) \Phi(k)= \\
& =\mathcal{U}^{(k)} p^{(k)}(t)\left(\mathcal{U}^{(k)}\right)^{-1} \Phi(k)=\left(p^{(0)}(t)+\nu(t) k\right) \Phi(k),
\end{aligned}
$$

что доказывает вторую из формул (89). Третья формула следует из уравнения Ланжевена (2) и уравнений (95), (96). Остается доказать первую формулу. При $\Phi \in \underset{\sim}{\mathcal{H}}$ и $k, k_{1} \in \mathbb{R}$ имеем

$$
\begin{aligned}
\left(e^{i k_{1} q_{w}} \Phi\right)(k) & =\left(\mathcal{W} e^{i k_{1} q_{w}} \mathcal{W}^{-1} \Phi\right)(k)=\pi^{(0 \leftarrow k)}\left(\mathcal{E}_{-k w}\right)\left(e^{i k_{1} q_{w}} \mathcal{W}^{-1}\right) \Phi(k)= \\
& =\pi^{(0 \leftarrow k)}\left(\mathcal{E}_{-k w}\right) \pi^{\left(k \leftarrow k-k_{1}\right)}\left(\mathcal{E}_{k_{1} w}\right) \pi^{\left(k-k_{1} \leftarrow 0\right)}\left(\mathcal{E}_{\left(k-k_{1}\right) w}\right) \Phi\left(k-k_{1}\right)= \\
& =\pi^{(0)}\left(\mathcal{E}_{-k w} \mathcal{E}_{k_{1} w} \mathcal{E}_{\left(k-k_{1}\right) w}\right) \Phi\left(k-k_{1}\right)=\pi^{(0)}\left(\mathcal{E}_{0}\right) \Phi\left(k-k_{1}\right)=e^{i k_{1} X} \Phi(k)
\end{aligned}
$$

Таким образом, получаем

$$
e^{i k_{1} q_{w}}=e^{i k_{1} X}, \quad \underset{\sim}{q_{w}}=X
$$

Поскольку из (88) следует, что $m \partial_{t} \underset{\sim}{q}(t)=\underset{\sim}{p}(t)$, то в силу соотношений (94) обе части первой из формул (89) имеют одну и ту же производную по $t$. Поэтому первая из формул (89) выполняется, если она выполняется при сглаживании хотя бы с одной основной функцией из $\mathcal{F}^{(1)}$. Учитывая (97), достаточно проверить, что

$$
q_{w}^{\mathrm{col}}=X, \quad \underset{\sim}{q} \stackrel{(0)}{w}=0
$$

Имеем:

$$
q_{w}^{\mathrm{col}}=\frac{K}{2 m} \int\left(\gamma^{2}-\partial_{t_{1}}^{2}\right) \varepsilon\left(t_{1}-t_{2}\right) \nu\left(t_{1}\right) \nu\left(t_{2}\right) d t_{1} d t_{2}+X \int w(t) d t=X
$$

Здесь использовано свойство антисимметричности по $t_{1}, t_{2}$ обобщенной функции $\left(\gamma^{2}-\partial_{t_{1}}^{2}\right) \varepsilon\left(t_{1}-t_{2}\right)$. Первое соотношение в (98) доказано. Второе соотношение в (98) следует из определения (93). Этим завершается доказательство первой из формул (89).

Сделаем два важных замечания. 
ЗАмЕчаниЕ 1. Вьше в (90) было дано конструктивное определение коллективной координаты, благодаря которому гильбертово пространство температурного представления и динамические переменные были приведены к достаточно простому виду (см. формулы (77), (89)). В этом замечании мы сформулируем согласованный набор условий, который позволяет "аксиоматически" определить коллективную координату с нужными свойствами, ограничившись случаем "классических" полей. Как и вьше, мы считаем фиксированной функцию $\nu(t)$ такую, что $2 \nu(t) / \gamma \in \mathcal{F}^{(1)}$. По ней определяются функции $w(t), \chi(t), \eta(t)$, как в $(74),(83),(84)$. Кроме того, по определению полагаем

$$
p^{\mathrm{col}}(t)=m \partial_{t} q^{\mathrm{col}}(t), \quad\left(\gamma+\partial_{t}\right) p^{\mathrm{col}}(t)=\sqrt{2 \gamma m} \xi^{\mathrm{col}}(t) .
$$

Первое условие заключается в том, что $q^{\mathrm{col}}(t)$ должно воспроизводить солитоноподобное поведение классического поля $q(t)$ при $t \rightarrow \pm \infty$. Поскольку (в силу (24))

$$
q(+\infty)-q(-\infty)=\frac{1}{m} \int p(t) d t=\frac{2}{\gamma m} K,
$$

то данное условие сводится к тому, что

$$
q^{\mathrm{col}}(+\infty)-q^{\mathrm{col}}(-\infty)=\frac{1}{m} \int p^{\mathrm{col}}(t) d t=\frac{2}{\gamma m} K .
$$

Очевидно, для этого достаточно положить

$$
p^{\mathrm{col}}(t)=\nu(t) K, \quad q^{\mathrm{col}}(t)=\chi(t) K+X
$$

где $X$ - не зависящая от времени динамическая переменная. Будем считать, что $X$ есть некий линейный функционал от координатного поля $q(t): X=q_{f}=\int q(t) f(t) d t$, $f(t) \in \mathcal{S}_{\mathrm{r}}(\mathbb{R})$. Второе условие для $q^{\mathrm{col}}(t)$ сводится к закону преобразования типа $(22)$ : $q^{\mathrm{col}}(t) \rightarrow q^{\mathrm{col}}(t)+r$ при пространственных трансляциях. Вместе с (22) это означает, что $\int f(t) d t=1$, т.е. $f(t) \in \mathcal{F}^{(1)}$. Третье условие заключается в том, что $q^{\mathrm{col}}(\tau)$ имеет нулевые скобки Пуассона (т.е. при квантовании коммутирует) с фононной составляюшей импульсного поля, определяемой как разность $p(t)-p^{\text {col }}(t)$. Это означает, что $\{p(t), X\}_{P}=\left\{p^{\mathrm{col}}(t), X\right\}_{P}$. Из перестановочного соотношения (5) и формулы (52) следует $2 \gamma\left(\gamma^{2}-\partial_{t}^{2}\right)^{-1} f(t)=\nu(t)$, откуда находим $f(t)=w(t)$, так что $X=q_{w}$.

Следовательно, три приведенных условия определяют на уровне классических полей коллективную координату $q^{\mathrm{col}}(t)$. Далее определяются фононные составляюшие так, чтобы вьполнялись разложения типа (89). Дальнейшее квантование в пространстве $\underset{\sim}{\mathcal{H}}(77)$ в соответствии с формулами $(80),(82)$ не представляет затруднений (это и есть упомянутый в разделе 1 альтернативный метод квантования). Заранее не очевидно, что полученные так представления ККС унитарно эквивалентны температурным представлениям из раздела 3 . То, что это так, доказано в данном разделе. 
ЗАмЕчАниЕ 2. Разложение (89), очевидно, зависит от выбора функции $\nu(t)$ или, эквивалентно, $\eta(t)$. При рассмотрении свойств конкретных состояний с матришами плотности $\rho$ в $\mathcal{H}$ иногда целесообразно выбирать $\eta(t)$ в зависимости от состояния. Если $\eta(t) K$ в (90) понимать как линейную однородную регрессию поля $\xi(t)$ на случайную величину $K$, то следует переопределить поле $\eta(t)$, заменив его выражением

$$
\eta^{(\rho)}(t)=\frac{\overline{\xi(t) K}}{\overline{K^{2}}} .
$$

Здесь и далее $\bar{A} \equiv \operatorname{tr}(\rho A)$ означает среднее значение наблюдаемой $A$ в состоянии с матрицей плотности $\rho$. Для достаточно широкого класса состояний, например, с матрицами плотности, являюшимися конечными линейными комбинациями операторов типа $\left|\psi_{1}\right\rangle\left\langle\psi_{2}\right| \otimes \sigma$, где $\psi_{j} \in \mathcal{S}_{\mathrm{r}}(\mathbb{R})$, а $\sigma$ - оператор вида (45), правая часть (99) (при прежнем произвольном выборе $\eta(t)$ ) обладает требуемым свойством гладкости, т.е. $\eta^{(\rho)}(t) \in$ $\mathcal{S}_{\mathrm{r}}(\mathbb{R})$. В качестве одного из применений формулы (99) отметим, что среднее значение гамильтониана (50) есть сумма отдельных вкладов переопределенного разложения $\underset{\sim}{\xi}(t)=\eta^{(\rho)}(t) K+\underset{\sim}{\xi^{(0)}}(t)$,

$$
\bar{H}=\frac{1}{2} \int\left(\eta^{(\rho)}(t)\right)^{2} \overline{K^{2}} d t+\frac{1}{2} \int \overline{N\left(\left({\underset{\sim}{(j)}(t)))^{2}}^{2}\right.\right.} d t
$$

\section{6. АСИМПТОТИЧЕСКИЕ СВОЙСТВА КОВАРИАЦИЙ}

Асимптотические свойства "свободного" классического броуновского процесса $q(t)$ при больших $t$ выражаются известной формулой Эйнштейна $\overline{q^{2}(t)} \sim 2 \mathcal{D} t$ при $t \rightarrow+\infty$, где $\mathcal{D}=(m \gamma \beta)^{-1}-$ константа дифффузии. При этом среднее значение $\overline{q(t)}$ постоянно.

Эволюция свободной квантово-механической частишы определяется формулами

$$
p(t)=p(0) \equiv P, \quad q(t)=q(0)+\frac{1}{m} p(0) t \equiv X+\frac{1}{m} P t .
$$

Поэтому зависимость от времени дисперсий и корреляционных моментов координаты и импульса может быть записана в явном виде:

$$
\begin{gathered}
D(p(t))=D(K), \quad D(q(t))=D(X)+\frac{2 t}{m} C_{+}(X, P)+\frac{t^{2}}{m^{2}} D(P), \\
C_{+}(p(t), q(t))=C_{+}(P, X)+\frac{t}{m} D(P), \quad C_{-}(p(t), q(t))=-\frac{i}{2} .
\end{gathered}
$$

Здесь мы используем обозначение $D(X)=\overline{X^{2}}-(\bar{X})^{2}$ для дисперсии оператора $X$ и

$$
C_{+}(X, Y)=\frac{1}{2} \operatorname{tr}\left(\rho[X-\bar{X}, Y-\bar{Y}]_{+}\right), \quad C_{-}(X, Y)=\frac{1}{2} \operatorname{tr}(\rho[X, Y])
$$


для симметризованного и антисимметризованного корреляционных моментов наблюдаемых $X, Y$ в состоянии с матрицей плотности $\rho$. Симметризованные и антисимметризованные корреляторы обнаруживают разное поведение со временем, поэтому их роль в соотношении неопределенностей

$$
\left|C_{+}(p, q)\right|^{2}+\left|C_{-}(p, q)\right|^{2} \leqslant D(p) D(q)
$$

различна (обсуждение соотношений неопределенностей в квантовых и диффузионных процессах см. в работах [15]).

Для “свободной” квантовой броуновской частицы мы докажем следующие асимптотические свойства при $t \rightarrow \infty$ :

$$
\begin{aligned}
D\left(p_{u}(t)\right) & \rightarrow\left\langle\left(p_{u}\right)^{2}\right\rangle, & D(q(t)) & \sim 2 \mathcal{D}|t|, \\
C_{+}(p(t), q(t)) & \rightarrow m \mathcal{D} \varepsilon(t), & C_{-}(p(t), q(t)) & =-\frac{i}{2} .
\end{aligned}
$$

Здесь и далее учитывается следующее обстоятельство. Координатное и импульсное поля $q(t)$ и $p(t)$ являются операторными обобщенными функциями. С помощью формулы (47) можно убедиться, что для последовательности $u_{n}(t) \in \mathcal{S}_{\mathrm{r}}(\mathbb{R})$, стремяшейся к $\delta(t)$ в $\mathcal{S}^{\prime}(\mathbb{R})$ при $n \rightarrow \infty$, существует предел для $e^{i l q_{u_{n}}(t)}$ при $n \rightarrow \infty$ (в слабой операторной топологии), определяющий оператор $e^{i l q(t)}$. Это есть следствие непрерывности функции $W(t)(32)$ при $t=0$. Тем самым определен оператор координаты $q(t)$ при фиксированном $t$.

В случае импульсного поля аналогичного предела для $e^{i l p_{u_{n}}}(t)$ не существует (это обусловлено отсутствием непрерывности $\partial_{t}^{2} W(t)$ при $t=0$; подобные ситуации носят обшее название ультрафиолетовых расходимостей). Таким образом, не сушествует оператора импульса $p(t)$ при фиксированном $t$. Тем не менее $p(t)$ и $N e^{i l p(t)}$ при фиксированном $t$ существуют как линейные функционалы на подходящем линейном подпространстве операторов с конечным следом. В качестве этого подпространства подходит линейная оболочка операторов указанного далее вида (102). Вообше, можно определить симметричные по $t_{1}, \ldots, t_{n}$ температурные нормальные произведения $N\left(q\left(t_{1}\right), \ldots, q\left(t_{n}\right)\right)$ с производящим функционалом $N E_{f}=E_{f} /\left\langle\mathcal{E}_{f}\right\rangle$ (аналогичным (41)). Как линейные функционалы на указанном подпространстве операторов они являются гладкими функциями по $t_{1}, \ldots, t_{n}$. Поэтому, например, соотношения (101) получаются формальной заменой $p_{u}(t)$ на $p(t)$ (в пределе, когда $\left.u(t) \rightarrow \delta(t),\left\langle\left(p_{u}\right)^{2}\right\rangle \rightarrow+\infty\right)$.

Поскольку дисперсии операторов сушествуют не для всех состояний, при выводе асимптотических соотношений следует ограничиться некоторым классом состояний. Как и в предыдушем разделе, мы будем считать, что температурное представление реализовано в пространстве $\underset{\sim}{\mathcal{H}}(77)$. Удобно выбрать класс состояний с матрицами плотности $\rho$ в $\underset{\sim}{\mathcal{H}}$, являющимися линейными комбинациями операторов конечного ранга вида

$$
\left|\psi_{1}\right\rangle\left\langle\psi_{2}\right| \otimes \sigma
$$


где $\psi_{j}(k) \in \mathcal{S}(\mathbb{R}), \sigma$ - оператор в $\mathcal{H}^{(0)}$ вида $(45)$. Обозначим посредством $\mathfrak{S}$ множество таких матриц плотности. (Произвольная матрица плотности может быть приближена с любой точностью по следовой норме операторами из $\mathfrak{S}$.)

Доказательство первого из соотношений (100) основывается на следуюшем свойстве импульсного поля: для него любое состояние с матрищей плотности $\rho$ в $\mathcal{H}$ при $t \rightarrow \infty$ стремится (по характеристическому функционалу) к гиббсовскому состоянию,

$$
\operatorname{tr}\left[\rho \exp \left(i \frac{l}{m} p_{\sim}(t)\right)\right] \rightarrow\left\langle\mathcal{E}_{f}\right\rangle=\exp \left(-\frac{l^{2}}{2 m}\left\langle\left(p_{u}\right)^{2}\right\rangle\right) \quad \text { при } \quad t \rightarrow \infty,
$$

где $f(t)=-\partial_{t} u(t), u(t) \in \mathcal{F}^{(1)}, l \in \mathbb{R}$. Достаточно убедиться, что при $t \rightarrow \infty$

$$
\operatorname{tr}\left[\left|\psi_{1}\right\rangle\left\langle\psi_{2}\right| \otimes \sigma \exp \left(i \frac{l}{m} p_{\sim}(t)\right)\right] \rightarrow\left\langle\mathcal{E}_{f}\right\rangle\left(\operatorname{tr}\left|\psi_{1}\right\rangle\left\langle\psi_{2}\right| \otimes \sigma\right) .
$$

Подставляя в (104) разложения (89) и учитывая соотношения

$$
\left\langle\psi_{2}\left|\exp \left(i \frac{l}{m} p_{u}^{\mathrm{col}}(t)\right)\right| \psi_{1}\right\rangle \rightarrow\left\langle\psi_{2} \mid \psi_{1}\right\rangle \quad \text { при } \quad t \rightarrow \infty,
$$

и (40), получаем (104) и (103). Соотношение (103) выполняется равномерно по $l$ в комплексной окрестности нуля. Дважды дифференцируя (103) по $l$, получаем $\overline{p_{u}(t)} \rightarrow 0$ при $t \rightarrow \infty$, а также первое из соотношений (100).

Для доказательства второго соотношения в (100) выведем формулу

$$
\operatorname{tr}\left[\rho \exp \left(i l|t|^{-1 / 2} \underset{\sim}{q}(t)\right)\right] \rightarrow \exp \left(-l^{2} \mathcal{D}\right) \quad \text { при } \quad t \rightarrow \infty
$$

Очевидно, коллективная составляющая $q^{\mathrm{col}}(t)(90)$ дает в пределе в (105) множитель 1. Остается рассмотреть в (105) вклад фононной составляюшей $q^{(0)}(t)$. Вначале рассмотрим состояние вида

$$
\rho=\rho^{\mathrm{col}} \otimes|\mathrm{GIBBS}\rangle\langle\mathrm{GIBBS}|,
$$

где $\rho^{\text {col }}$ - матрища плотности в $\mathcal{L}^{2}(\mathbb{R}, d k / 2 \pi)$. Тогда в силу $(93),(37),(38)$ левая часть $(105)$ с $\underset{\sim}{q}{ }^{(0)}(t)$ вместо $\underset{\sim}{q}(t)$ есть

$$
\begin{aligned}
& \exp \left(-l^{2}|t|^{-1} \frac{\gamma}{m} \int\left(\frac{1-e^{-\beta \lambda}}{\lambda}\right)^{-1} \frac{1}{\gamma^{2}+\lambda^{2}}\left|e^{i \lambda t}-\widetilde{w}(\lambda)\right|^{2} P \frac{1}{\lambda^{2}} \frac{d \lambda}{2 \pi}\right)= \\
& \quad=\exp \left(-l^{2}|t|^{-1} \frac{2 \gamma}{m}\left(\operatorname{Re} a_{1}(t)-a_{1}(0)\right)\right)
\end{aligned}
$$

где

$$
a_{1}(t)=-\int\left(\frac{1-e^{-\beta \lambda}}{\lambda}\right)^{-1} \frac{1}{\gamma^{2}+\lambda^{2}} e^{-i \lambda t} \widetilde{w}(\lambda) P \frac{1}{\lambda^{2}} \frac{d \lambda}{2 \pi}
$$


Отсюда следует, что $\partial_{t} a_{1}(t)=(1 / 2) \varepsilon(t) * a_{2}(t)$, где $a_{2}(t) \in \mathcal{S}_{\mathrm{r}}(\mathbb{R})$ и $\tilde{a}_{2}(0)=\left(\beta \gamma^{2}\right)^{-1}$. Поэтому $\partial_{t} a_{1}(t)=\left(2 \beta \gamma^{2}\right)^{-1} \varepsilon(t)+a_{3}(t)$, где $a_{3}(t)-$ быстро убываюшая функция при $t \rightarrow \infty$. В результате имеем $a_{1}(t)=\left(2 \beta \gamma^{2}\right)^{-1}|t|+a_{4}(t)$, где $a_{4}(t)$ - ограниченная функция. Согласно формуле (107) в этом случае предельное соотношение (105) выполнено.

Остается проверить соотношение (105) для матриц $\rho \in \mathfrak{S}$. Как и при доказательстве сотношения (40), достаточно доказать следуюший аналог предела (44) (где $f_{j}=-\partial_{t} g_{j}$, $g_{j} \in \mathcal{S}_{\mathrm{r}}(\mathbb{R})$ и $q^{(0)}(t)$ выражается через $p^{(0)}(t)$ формулой типа $\left.(92)\right)$ :

$$
\begin{gathered}
\left\langle\text { GIBBS }\left|N E_{f_{1}}^{(0)} N \exp \left(i l|t|^{-1 / 2} q^{(0)}(t)\right) N E_{f_{2}}^{(0)}\right| \mathrm{GIBBS}\right\rangle_{0} \rightarrow \\
\rightarrow\left\langle\operatorname{GIBBS}\left|N E_{f_{1}}^{(0)} N E_{f_{2}}^{(0)}\right| \mathrm{GIBBS}\right\rangle_{0} \quad \text { при } \quad t \rightarrow \infty,
\end{gathered}
$$

так как величина $\exp \left(i l|t|^{-1 / 2} q^{(0)}(t)\right)$ отличается от $N \exp \left(i l|t|^{-1 / 2} q^{(0)}(t)\right)$ множителем $\left\langle\exp \left(i l|t|^{-1 / 2} q^{(0)}(t)\right)\right\rangle$, совпадающим с (107) и по доказанному стремящимся к $\exp \left(-l^{2} \mathcal{D}\right)$ при $t \rightarrow \infty$. Используя соотношения $(42),(43)$, нетрудно убедиться, что левая часть в (109) отличается от правой множителями, стремящимися к 1 при $t \rightarrow \infty$. Тем самым доказана формула (109), а значит, и (105). Учитывая равномерность предела (105) по $l$ в комплексной окрестности нуля, получаем дифференцированием по $l$, что $\left.|t|^{-1 / 2} \operatorname{tr} \underset{\sim}{\rho q}(t)\right) \rightarrow 0$ при $t \rightarrow \infty$, а также второе из соотношений (100).

Доказательство первой из формул (101) основывается на соотношении

$$
\begin{aligned}
& \operatorname{tr}\left(\rho N \exp \left(i l_{1}{\underset{\sim}{\sim}}^{(0)}(t) N \exp \left(i l_{2}{\underset{\sim}{q}}^{(0)}(t)\right)\right) \rightarrow\right. \\
& \quad \rightarrow \exp \left(-l_{1} l_{2}(\langle p(0) q(0)\rangle+m \mathcal{D} \varepsilon(t))\right) \quad \text { при } \quad t \rightarrow \infty .
\end{aligned}
$$

Сначала докажем (110) для $\rho$ вида (106). В силу (93), (42), (43) левую часть (109) можно записать как

$$
\begin{aligned}
& \exp \left(l_{1} l_{2} \int\left(\frac{1-e^{-\beta \lambda}}{\lambda}\right)^{-1} \frac{1}{\gamma^{2}+\lambda^{2}} 2 i P \frac{1}{\lambda}\left(e^{i \lambda t}-\widetilde{w}(\lambda)\right) \frac{d \lambda}{2 \pi}\right)= \\
& \quad=\exp \left(-l_{1} l_{2}\left(\langle p(0) q(0)\rangle+2 \gamma \partial_{t} a_{1}(t)\right)\right)
\end{aligned}
$$

где $a_{1}(t)$ - функция, определенная формулой (108). Как было установлено ранее, $\left(\partial_{t} a_{1}(t)-\left(2 \beta \gamma^{2}\right)^{-1} \varepsilon(t)\right) \rightarrow 0$ при $t \rightarrow \infty$. Следовательно, из формулы (111) вытекает (110) при спешиальном выборе $\rho$ в виде (106). Затем, как и при выводе (109), устанавливается (110) для произвольной матрицы плотности $\rho \in \mathfrak{S}$. Дифференцирование (110) по $l_{1}$ и $l_{2}$ в нуле дает

$$
\operatorname{tr}\left(\rho p^{p}{ }^{(0)}(t) \underset{\sim}{q(0)}(t)\right) \rightarrow(\langle p(0) q(0)\rangle+m \mathcal{D} \varepsilon(t)) \quad \text { при } \quad t \rightarrow \infty .
$$

Используя разложения (89), нетрудно отсюда получить, что

$$
\operatorname{tr}(\rho \underset{\sim}{p}(t) \underset{\sim}{q}(t)) \rightarrow(\langle p(0) q(0)\rangle+m \mathcal{D} \varepsilon(t)) \quad \text { при } \quad t \rightarrow \infty .
$$


В результате имеем

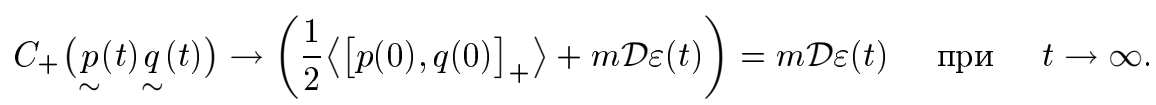

Тем самым доказано первое из соотношений (101). Второе из соотношений (101) есть следствие формулы (5).

Для характеристики квантовых процессов при так называемых малых временах (или мальх расстояниях) применим метод разложения операторного произведения (operator product expansion, OPE), хотя в данном случае наряду с операторами фигурируют полуторалинейные формы. Имеет место следуюшее асимптотическое разложение при $t \rightarrow 0$ (где $A$ - некоторая вешественная константа):

$$
\begin{aligned}
{[q(t), q(0)]_{+}=} & (q(0))^{2}+\frac{t}{m}[p(0), q(0)]_{+}+\frac{t^{2}}{2 m} N\left(q(0) \partial_{t} p(0)\right)- \\
& -\frac{\gamma t^{2}}{2 \pi m}(\ln (\gamma|t|)+A)+o\left(t^{2}\right)
\end{aligned}
$$

Для доказательства воспользуемся температурными нормальными произведениями координатного поля с производящим функционалом $N E_{f}=E_{f}\left\langle\mathcal{E}_{f}\right\rangle^{-1}$ (это определение аналогично (41)). Тогда

$$
[q(t), q(0)]_{+}=N(q(t) q(0))+\left\langle[q(t), q(0)]_{+}\right\rangle=N(q(t) q(0))+\operatorname{Re} W(t) .
$$

Аналогично найдем $(q(0))^{2}=N(q(0))^{2}+W(0)$. В результате

$$
[q(t), q(0)]_{+}=(q(0))^{2}+N(q(0)(q(t)-q(0)))+\operatorname{Re}(W(t)-W(0)) .
$$

Согласно (32) имеем

$$
\operatorname{Re} W(t)=-\frac{1}{2 \pi m} \ln \left(\varkappa \frac{\beta}{\pi} \operatorname{sh} \frac{\pi|t|}{\beta}\right) e^{-\gamma|t|}=R_{1}(t)+R_{2}(t)
$$

где

$$
\begin{aligned}
& R_{1}(t)=-\frac{1}{2 \pi m} \ln (\varkappa|t|) e^{-\gamma|t|}, \\
& R_{2}(t)=-\frac{1}{2 \pi m} \ln \left(\frac{\beta}{\pi t} \operatorname{sh} \frac{\pi t}{\beta}\right) e^{-\gamma|t|},
\end{aligned}
$$

причем $R_{2}(t)$ - вещественная четная функция класса $\mathcal{C}^{\infty}$. Отсюда получаем

$$
\partial_{t} R_{1}(t)=-\frac{1}{2 \pi m} \int P \frac{1}{t-\tau} e^{-\gamma|\tau|} d \tau=-\frac{1}{2 \pi m}\left(e^{-\gamma t} \operatorname{Ei}(\gamma t)-e^{\gamma t} \operatorname{Ei}(-\gamma t)\right) .
$$

Используя поведение интегральной показательной функции ([16], формулы 8.214.1-2)

$$
\operatorname{Ei}(x) \equiv e^{x}\left(P \frac{1}{x}\left(\theta(x) e^{-x}\right)\right)=\ln |x|+C+x+x^{2} a(x),
$$


где $C$ - константа Эйлера, $a(x)$ - некоторая функция класса $\mathcal{C}^{\infty}$, получаем

$$
\partial_{t} R_{1}(t)=\frac{\gamma t}{\pi m}(\ln (\gamma|t|)+C-1)+t^{2}\left(\ln (\gamma|t|) a_{1}(t)+a_{2}(t)\right),
$$

где $a_{1}(t), a_{2}(t)$ - гладкие функции. Следовательно,

$$
R_{1}(t)=a_{0}+\frac{\gamma}{2 \pi m} t^{2}\left(\ln (\gamma|t|)+C-\frac{3}{2}\right)+o\left(t^{2}\right) \quad \text { при } \quad t \rightarrow 0
$$

где $a_{0}$ - некоторая константа. Последнее соотношение вместе с формулами (113)-(115) доказывает разложение (112).

Из (112) вытекает формула для корреляторов, а также аналогичные формулы с участием импульсного поля (которые доказываются по той же схеме),

$$
\begin{aligned}
C_{+}(q(t), q(0))= & D(q(0))+\frac{t}{m} C_{+}(p(0), q(0))+\frac{t^{2}}{2 m} N C_{+}\left(q(0), \partial_{t} p(0)\right)- \\
& -\frac{\gamma t^{2}}{2 \pi m}(\ln (\gamma|t|)+A)+o\left(t^{2}\right), \\
C_{+}(p(t), q(0))= & C_{+}(p(0), q(0))+t N C_{+}\left(q(0), \partial_{t} p(0)\right)- \\
& -\frac{\gamma t}{\pi m}\left(\ln (\gamma|t|)+A-\frac{1}{2}\right)+o\left(t^{2}\right), \\
C_{+}(q(t), p(0))= & C_{+}(p(0), q(0))+t N D\left((p(0))^{2}\right)+ \\
& +\frac{\gamma t}{\pi m}\left(\ln (\gamma|t|)+A-\frac{1}{2}\right)+o\left(t^{2}\right) .
\end{aligned}
$$

Здесь использованы обозначения

$$
N D(X)=\overline{N\left(X^{2}\right)}-(\bar{X})^{2}, \quad N C_{+}(X, Y)=\overline{N(X Y)}-\bar{X} \bar{Y}
$$

Формулы (100), (101) показывают, что при $t \rightarrow \infty$ воспроизводятся асимптотические свойства корреляторов классической “свободной” броуновской частицы. При $t \rightarrow 0$ уместно сравнение формулы (112) с соответствующей формулой для свободной квантово-механической частицы

$$
[q(t), q(0)]_{+}=(q(0))^{2}+\frac{t}{m}[p(0), q(0)]_{+} .
$$




\section{7. БРОУНОВСКОЕ ДВИЖЕНИЕ КВАНТОВОГО ОСЦИЛЛЯТОРА}

Уравнение Ланжевена для броуновского движения одномерного гармонического осциллятора имеет вид

$$
m\left(\partial_{t}+\gamma\right) \partial_{t} Q(t)+m \omega^{2} Q(t)=\sqrt{2 \gamma m} \Xi(t)
$$

Здесь $Q(t)$ - координата, $m \partial_{t} Q(t)=P(t)$ - импульс частицы; правая часть в $(120)$ есть флуктуируюшая сила. Как и в разделе 2 , для вывода $\mathrm{KKC}$ координатного поля $Q(t)$ мы привлекаем струнную модель. Отличие сводится к тому, что на точечную частицу, прикрепленную к концу $(x=0)$ полубесконечной струны, дополнительно действует сила $-m \omega^{2} Q(t)$ вдоль оси $O y$. Соответственно новый струнный лагранжиан получается из прежнего вычитанием потенциала

$$
\frac{1}{2} m \omega^{2}(Q(t))^{2}=\frac{\omega^{2}}{2 \gamma} \int(\Phi(t, x))^{2} \delta(x) d x
$$

Здесь $\Phi(t, x)$ - струнное поле, через которое координата частицы выражается формулой вида (8):

$$
Q(t)=(\gamma m)^{-1 / 2} \Phi(t, 0) .
$$

Лагранжевы уравнения движения сводятся к волновому уравнению $\left(\partial_{t}^{2}-\partial_{x}^{2}\right) \Phi \theta(x)=0$ и граничному условию при $x=0:\left(\partial_{t}^{2}+\omega^{2}-\gamma \partial_{x}\right) \Phi \delta(x)=0$. Решение волнового уравнения по-прежнему записывается в виде

$$
\Phi(t, x)=\frac{1}{\sqrt{2}}\left(\Phi^{\mathrm{in}}(t+x)+\Phi^{\mathrm{out}}(t-x)\right) .
$$

В случае "классического" поля $\Phi(t, x)$ мы полагаем, что это вещественная функция класса $\mathcal{C}^{\infty}$ от $(t, x) \in \mathbb{R} \times \overline{\mathbb{R}}_{+}$, причем $\partial_{x} \Phi$ и $\partial_{t} \Phi$ принадлежат $\mathcal{S}_{\mathrm{r}}(\mathbb{R})$ по $x$ при любом $t^{4)}$. Множество таких решений уравнений движения образует фазовое симплектическое пространство $\mathcal{X}$. Можно показать, что входящее и выходящие поля связаны соотношением

$$
\Phi^{\text {out }}(t)=\frac{\gamma \partial_{t}-\partial_{t}^{2}-\omega^{2}}{\gamma \partial_{t}+\partial_{t}^{2}+\omega^{2}} \Phi^{\text {in }}(t)
$$

а их производные по $t$ принадлежат пространству $\mathcal{S}_{\mathrm{r}}(\mathbb{R})$. Из формул (121)-(123) следует выражение для координатного поля

$$
Q(t)=\left(\frac{2 \gamma}{m}\right)^{1 / 2} \frac{1}{\gamma \partial_{t}+\partial_{t}^{2}+\omega^{2}} \partial_{t} \Phi^{\mathrm{in}}(t)
$$

\footnotetext{
${ }^{4)}$ В работе [7] налагалось более сильное ограничение: $\Phi(t, x)$ и $\partial_{t} \Phi(t, x)$ принадлежат $\mathcal{S}_{\mathrm{r}}(\mathbb{R})$ по $x$. Тем самым исключались солитоноподобные конфигурации поля $\Phi^{\text {in }}(t)$ и топологический заряд $Z$ модели оказывался тождественно равным нулю.
} 
$\left(\right.$ Отметим, что $Q(t)$ также принадлежит $\mathcal{S}_{\mathrm{r}}(\mathbb{R})$.) Чтобы выполнялось уравнение Ланжевена (12), следует отождествить поле $\Xi(t)$ с входяшим током $J^{\text {in }}(t)$, т.е.

$$
\Xi(t)=J^{\mathrm{in}}(t) \equiv \partial_{t} \Phi^{\mathrm{in}}(t) .
$$

По заданному полю $\Phi(t, x)$ поле $\Phi^{\text {in }}(t)$ определяется разложением $(122)$ с точностью до константы. Для однозначности разложения наложим калибровочное условие

$$
\Phi^{\text {in }}(+\infty)+\Phi^{\text {in }}(-\infty)=0 .
$$

В этом случае поле $\Phi^{\text {in }}(t)$ является произвольной функцией класса $\varepsilon * \mathcal{S}_{\mathrm{r}}(\mathbb{R})$ и служит способом параметризации симплектического пространства ${ }^{5)}$ с симплектической формой

$$
\sigma\left(\Phi_{1}^{\mathrm{in}}, \Phi_{2}^{\mathrm{in}}\right)=\frac{1}{2} \int\left(\left(\partial_{t} \Phi^{\mathrm{in}}(t)\right) \Phi_{2}^{\mathrm{in}}(t)-\Phi_{1}^{\mathrm{in}}(t) \partial_{t} \Phi_{2}^{\mathrm{in}}(t)\right) d t .
$$

Формула

$$
\left\{\sigma\left(\Phi^{\mathrm{in}}, \Phi_{1}^{\mathrm{in}}\right), \sigma\left(\Phi^{\mathrm{in}}, \Phi_{2}^{\mathrm{in}}\right)\right\}_{P}=\sigma\left(\Phi_{1}^{\mathrm{in}}, \Phi_{2}^{\mathrm{in}}\right)
$$

при $\Phi_{1}, \Phi_{2} \in \mathcal{X}, \Phi \in \widetilde{\mathcal{X}}$ позволяет найти разновременную скобку Пуассона поля $\Phi^{\text {in }}$, которая совпадает с (14),

$$
\left\{\Phi^{\mathrm{in}}\left(t_{1}\right), \Phi^{\mathrm{in}}\left(t_{2}\right)\right\}_{P}=D^{\mathrm{in}}\left(t_{1}-t_{2}\right) .
$$

Гамильтониан $H$ в терминах $\Phi^{\text {in }}$ имеет вид

$$
H=\frac{1}{2} \int\left(J^{\text {in }}(t)\right)^{2} d t,
$$

а топологический заряд

$$
Z=\frac{1}{\sqrt{2}}\left(\Phi^{\mathrm{in}}(+\infty)-\Phi^{\mathrm{in}}(-\infty)\right)=\frac{1}{\sqrt{2}} \int J^{\mathrm{in}}(t) d t .
$$

Заряд $Z$ является генератором калибровочных преобразований

$$
\Phi^{\mathrm{in}}(t) \rightarrow \Phi^{\mathrm{in}}(t)+\frac{1}{\sqrt{2}} g, \quad g \in \mathbb{R} .
$$

Из соотношения (123) следует, что

$$
\Phi^{\text {out }}(t) \rightarrow \Phi^{\text {out }}(t)-\frac{1}{\sqrt{2}} g,
$$

\footnotetext{
5) При отбрасывании калибровочного условия $\Phi^{\text {in }}(+\infty)+\Phi^{\text {in }}(-\infty)=0$ соответствующие конфигурации поля $\Phi^{\text {in }}(t)$ образуют расширение $\widetilde{\mathcal{X}}$ пространства $\mathcal{X}$. $\widetilde{\mathcal{X}}$ является двойственным к $\mathcal{X}$

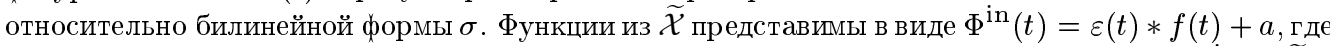
$f(t) \in \mathcal{S}_{\mathrm{r}}(\mathbb{R}), a \in \mathbb{R}$. Полевая алгебра классического поля состоит из функционалов от $\Phi^{\text {in }} \in \widetilde{\mathcal{X}}$.
} 
так что поля $\Phi(t, x), J^{\text {in }}(t), J^{\text {out }}(t), Q(t), P(t)$ являются калибровочно-инвариантными; мы назовем их наблюдаемыми полями.

В данном случае отсутствует инвариантность системы относительно пространственных трансляций и аналог формулы $(23) K=(\gamma m)^{1 / 2} Z$ не интерпретируется как полный импульс системы. Выясним смысл $Z$ и $K$ в терминах наблюдаемых полей. Из определения

$$
Z=\frac{1}{\sqrt{2}}\left(\Phi^{\mathrm{in}}(+\infty)-\Phi^{\mathrm{in}}(-\infty)\right)=\frac{1}{\sqrt{2}}\left(\Phi^{\mathrm{in}}(+\infty)+\Phi^{\mathrm{out}}(-\infty)\right)
$$

следует, что $Z=\Phi(t,+\infty)$. Из формул $(125),(128)$ получаем

$$
2 K=\int \sqrt{2 \gamma m} \Xi(t) d t=\int m \omega^{2} Q(t) d t
$$

так что $2 K$ представляет собой импульс, переданный частице флуктуирующей силой за время от $-\infty$ до $+\infty$, a $-2 K-$ импульс, переданный частице упругой силой за время от $-\infty$ до $+\infty$.

Сравнение формул (126), (127) с формулами (14), (18) показывает, что поля $\Phi^{\text {in }}(t)$ и $\varphi^{\text {in }}(t)$ как гамильтоновы системы эквивалентны, поэтому имеет смысл отождествить их,

$$
\Phi^{\mathrm{in}}(t)=\varphi^{\mathrm{in}}(t) .
$$

Соответственно будем иметь

$$
J^{\text {in }}(t) \equiv \Xi(t)=\xi(t) \equiv j^{\text {in }}(t)
$$

В результате из формул (124) и (111) (или из (12) и (2)) получаем следующее выражение координатного поля $Q(t)$ броуновского осциллятора через координатное $q(t)$ или импульсное $p(t)$ поле “свободной” броуновской частицы:

$$
\begin{aligned}
Q(t) & =\frac{\left(\gamma+\partial_{t}\right) \partial_{t}}{\gamma \partial_{t}+\partial_{t}^{2}+\omega^{2}} q(t) \\
Q(t) & =\frac{1}{m} \frac{\gamma+\partial_{t}}{\gamma \partial_{t}+\partial_{t}^{2}+\omega^{2}} p(t)
\end{aligned}
$$

Обратно, импульсное поле $p(t)$ может быть выражено через $Q(t)$. Координатное поле $q(t)$ через $Q(t)$ однозначно не выражается. Алгебра поля $Q(t)$ совпадает с импульсной подалгеброй поля $q(t)$, состояшей из инвариантов пространственных трансляций $(22)$.

Из (124) и коммутатора (16) следуют ККС поля

$$
\left[Q\left(t_{1}\right), Q\left(t_{2}\right)\right]=\frac{2 \gamma}{m} \int \frac{\lambda}{(\gamma \lambda)^{2}+\left(\lambda^{2}-\omega^{2}\right)^{2}} e^{-i \lambda\left(t_{1}-t_{2}\right)} \frac{d \lambda}{2 \pi}
$$

6 Теоретическая и математическая физика, т. 136, № 1, 2003 г. 
Считая $q(t), p(t)$ в (133) или (134) полями в температурном представлении $\pi$, получаем температурное представление координатного поля $Q(t)$ квантового броуновского осциллятора. В соответствии с формулой (70) оно разлагается в прямой интеграл представлений $\pi^{(k)}$ в обобщенных подпространствах $\mathcal{H}^{(k)}$ с определенными значениями оператора $K$ ( $K$ принадлежит к центру алгебры поля $Q(t)$ и поэтому играет роль суперотборного оператора). Представление $\pi^{(0)}$ является гиббсовским представлением с циклическим вектором $\mid$ GIBBS $\rangle$ стационарного гиббсовского состояния $s^{(0)}$ и с двухточечной функцией (получаемой из (38)):

$$
\begin{aligned}
& \left\langle\operatorname{GIBBS}\left|Q^{(0)}\left(t_{1}\right) Q^{(0)}\left(t_{2}\right)\right| \mathrm{GIBBS}\right\rangle_{0} \equiv\left\langle Q\left(t_{1}\right) Q\left(t_{2}\right)\right\rangle= \\
& \quad=\frac{2 \gamma}{m} \int\left(\frac{1-e^{-\beta \lambda}}{\lambda}\right)^{-1} \frac{1}{(\gamma \lambda)^{2}+\left(\lambda^{2}-\omega^{2}\right)^{2}} e^{-i \lambda\left(t_{1}-t_{2}\right)} \frac{d \lambda}{2 \pi} .
\end{aligned}
$$

Представления $\pi^{(k)}$ попарно унитарно неэквивалентны и являются смешенными гиббсовскими представлениями.

Разбиению (89) соответствует выражение поля $\underset{\sim}{Q}(t)$ в $\underset{\sim}{\mathcal{H}}(77)$ в виде суммы "классической” $Q^{\mathrm{cl}}(t)$ и фононной $\underset{\sim}{Q^{(0)}}(t)$ составляюших (которые взаимно коммутируют),

$$
\underset{\sim}{Q}(t)=Q^{\mathrm{cl}}(t)+\underset{\sim}{Q^{(0)}}(t) \equiv N(t) K+1 \otimes Q^{(0)}(t)
$$

Здесь

$$
N(t)=\frac{1}{m} \frac{\gamma+\partial_{t}}{\gamma \partial_{t}+\partial_{t}^{2}+\omega^{2}} \nu(t), \quad \frac{m \omega^{2}}{2} N(t) \in \mathcal{F}^{(1)} .
$$

Асимптотические свойства корреляций полей $Q(t)$ и $P(t)$ при $t \rightarrow \infty$ аналогичны свойству импульсного поля $p(t)$, выраженному первым из соотношений $(100)$,

$$
\begin{gathered}
D(\underset{\sim}{P} u(t)) \rightarrow\left\langle\left(P_{u}\right)^{2}\right\rangle, \quad D(\underset{\sim}{Q}(t)) \rightarrow\left\langle(Q(0))^{2}\right\rangle, \\
C_{+}(\underset{\sim}{P}(t), \underset{\sim}{Q}(t)) \rightarrow\left\langle[P(0), Q(0)]_{+}\right\rangle=0 .
\end{gathered}
$$

Доказательство, как и вывод соотношения (103), основано на свойстве (40) сильного перемешивания гиббсовского состояния $s^{(0)}$. Также для поля $Q(t)$ имеет место асимптотическое соотношение типа (112) при $t \rightarrow 0$ (с другой константой $A)$.

Итак, в данном разделе мы построили более широкое, чем стандартное [7], представление квантового броуновского осциллятора. Это представление все еше может считаться температурным, поскольку броуновский осциллятор реализован как часть другой системы, а именно “свободной” броуновской частицы в температурном представлении, которое, в свою очередь, построено с помошью стационарного обобшенного состояния с определенной температурой.

В заключение отметим, что данная работа выполнена в рамках складывающегося направления трактовки квантовых случайных процессов с позиций гамильтоновой теории 
поля. Мы показываем уместность и эффективность подобного подхода к "свободной" квантовой броуновской частице, подчиняюшейся уравнению Ланжевена. Если в работе [10] было сформулировано понятие температурного представления динамических переменных такой частишы, то здесь развит технический аппарат для работы с ним. Для этой цели наиболее приспособлена концепция коллективной координаты. Во-первых, она нашла широкое применение в квантовой теории поля и статистической физике как способ обойти трудности с инфракрасными расходимостями и максимально приспособить традиционные методы к ситуациям с представлениями, далекими от фоковских или гиббсовских представлений свободных полей. Во-вторых, коллективная координата имеет ясный физический смысл, выявляя асимптотику полей на пространственно-временной бесконечности.

\section{Список литературы}

[1] W. H. Louisell. Radiation and Noise in Quantum Electronics. New York: McGraw-Hill, 1965.

[2] W. H. Louisell. Quantum Statistical Properties of Radiation. New York: Wiley, 1973.

[3] Я.Б. Зельдович, А. М. Переломов, В.С. Попов.ЖЭТФ. 1968. Т. 55. С. 589; 1969. T. 57. C. 196.

[4] A. O. Caldeira, A. J. Leggett. Ann. Phys. 1983. V. 149. P. 34; 1984. V. 153. P. 415.

[5] L. Accardi, Y. G. Lu, I. V. Volovich. Quantum Theory and Its Stochastic Limit. Berlin: Springer, 2002.

[6] G. W. Ford, M. Kac, P. Mazur. J. Math. Phys. 1965. V. 6. P. 504.

[7] J. T. Lewis, H. Maasen. Hamiltonian models of classical and quantum stochastic processes. In: Quantum Probability and Applications to the Quantum Theory of Irreversible Processes. Proc. of the Int. Workshop (Villa Mondragone, Italy, September 6-11, 1982). Lect. Notes in Math. V. 1055. Eds. L. Accardi, A. Frigerio, V. Giorini. Berlin: Springer, 1984. P. 245.

[8] H. Dekker. Phys. Rep. 1981. V. 80. P. 1.

[9] H. Lamb. Proc. London Math. Soc. 1900. V. 2. P. 88.

[10] А. И. Оксак, А. Д. Суханов. ТМФ. 1998. Т. 116. С. 201.

[11] А. И. Оксак. ТМФ. 1981. Т. 48. С. 297.

[12] Н. Н. Боголюбов, А. А. Логунов, А. И. Оксак, И. Т. Тодоров. Общие принципы квантовой теории поля. М.: Наука, 1987.

[13] Р. Раджсараман. Солитоны и инстантоны в квантовой теории поля. М.: Мир, 1985.

[14] Ж. Диксмье. С* -алгебры и их представления. М.: Наука, 1974.

[15] А.Д. Суханов. ЭЧАЯ. 2001. Т. 32. С. 1177; ТМФ. 2002. Т. 132. С. 449.

[16] И. С. Градитейн, Г. М. Рыжик. Таблицы интегралов, сумм, рядов и произведений. М.: Физматгиз, 1963. 